\title{
The pseudokinase MLKL mediates programmed hepatocellular necrosis independently of RIPK3 during hepatitis
}

\author{
Claudia Günther, ${ }^{1}$ Gui-Wei He, ${ }^{1}$ Andreas E. Kremer, ${ }^{1}$ James M. Murphy, ${ }^{2,3}$ Emma J. Petrie, ${ }^{2,3}$ Kerstin Amann, ${ }^{4}$ \\ Peter Vandenabeele, ${ }^{5,6}$ Andreas Linkermann, ${ }^{7}$ Christopher Poremba, ${ }^{8}$ Ulrike Schleicher, ${ }^{9}$ Christin Dewitz, ${ }^{7}$ \\ Stefan Krautwald, ${ }^{7}$ Markus F. Neurath, ${ }^{1}$ Christoph Becker, ${ }^{1}$ and Stefan Wirtz ${ }^{1}$
}

\begin{abstract}
'Department of Medicine, Friedrich-Alexander-University, Erlangen, Germany. ${ }^{2}$ Walter and Eliza Hall Institute of Medical Research, Parkville, Victoria, Australia. ${ }^{3}$ Department of Medical Biology, University of Melbourne, Parkville, Victoria, Australia. ${ }^{4}$ Department of Nephropathology, Friedrich-Alexander-University, Erlangen, Germany. ${ }^{5}$ Department of Biomedical Molecular Biology, Chent University, Chent, Belgium. ${ }^{6}$ Inflammation Research Center, VIB, Chent, Belgium. ${ }^{7}$ Department of Nephrology and Hypertension, University Hospital Schleswig-Holstein, Kiel, Germany. ${ }^{8}$ Department of Pathology, Pathology Munich-North, Munich, Germany. ${ }^{9}$ Mikrobiologisches Institut, Universitätsklinikum Erlangen, Erlangen, Germany.
\end{abstract}

\begin{abstract}
Although necrosis and necroinflammation are central features of many liver diseases, the role of programmed necrosis in the context of inflammation-dependent hepatocellular death remains to be fully determined. Here, we have demonstrated that the pseudokinase mixed lineage kinase domain-like protein (MLKL), which plays a key role in the execution of receptorinteracting protein (RIP) kinase-dependent necroptosis, is upregulated and activated in human autoimmune hepatitis and in a murine model of inflammation-dependent hepatitis. Using genetic and pharmacologic approaches, we determined that hepatocellular necrosis in experimental hepatitis is driven by an MLKL-dependent pathway that occurs independently of RIPK3. Moreover, we have provided evidence that the cytotoxic activity of the proinflammatory cytokine IFN- $\gamma$ in hepatic inflammation is strongly connected to induction of MLKL expression via activation of the transcription factor STAT1. In summary, our results reveal a pathway for MLKL-dependent programmed necrosis that is executed in the absence of RIPK3 and potentially drives the pathogenesis of severe liver diseases.
\end{abstract}

\section{Introduction}

Although the liver is an organ of remarkable regenerative capacity, cell death-related compensatory tissue injury responses commonly culminate in fibrosis and eventually cirrhosis, a major cause of morbidity worldwide. With regard to this vital contribution of hepatocellular death to virtually all hepatic diseases, precise mechanistic knowledge of cell death regulation is essential to understand the pathophysiology of liver diseases. While for a long time apoptosis and necrosis were the most widely recognized forms of cell death, the concept of regulated cell death was recently challenged by the discovery of necroptosis $(1,2)$. Necroptosis has been described as a form of cell death mediated by the receptor-interacting protein kinase RIPK3 and mixed lineage kinase domain-like protein (MLKL) that is sensitized under certain conditions, such as caspase-8 inhibition (3-8). In the absence of functional caspase-8, receptor-interacting protein kinases (RIP kinases) drive the assembly of a macromolecular complex, the so-called necrosome (9). It is currently believed that necrosome formation is a critical step during necroptosis, as it leads

\section{Related Commentary: doi:10.1172/JCI90830}

Authorship note: C. Becker and S. Wirtz are co-senior authors.

Conflict of interest: The authors have declared that no conflict of interest exists. Submitted: March 14, 2016; Accepted: September 1, 2016.

Reference information: J Clin Invest. 2016;126(11):4346-4360. doi:10.1172/JCI87545. to recruitment and activation of the RIPK3 substrate MLKL (7). Activated MLKL subsequently forms oligomers and translocates to the plasma membrane and other membranous cellular structures to cause membrane disintegration, a critical step required for cell death execution $(10,11)$. While apoptosis is considered to be rather immunosuppressive, necroptosis has been suggested to be proinflammatory and to initiate inflammation. Accordingly, studies implicated necroptosis in the pathogenesis of several inflammatory diseases, such as inflammatory bowel disease and kidney diseases $(8,12-14)$. Conversely, the role of programmed necrotic cell death in human inflammatory liver diseases still remains to be fully elucidated (15). In patients suffering from drug-induced liver injury (DILI), cell death was demonstrated to be associated with activation of MLKL (16). However, the role of programmed hepatocellular death in acetaminophen-induced (APAP-induced) murine liver damage remains controversial. Although inhibition of necroptosis by deficiency of RIPK3 (17) or pharmacological blockage of RIPK1 kinase activity (18) reduced cell death at early time points during APAP-induced hepatic injury, RIPK3 and MLKL deficiency was unable to prevent liver injury in this model (19). Other studies further demonstrated that ethanol-induced hepatic injury is independent of RIPK1 kinase activity but dependent on RIPK3, suggesting that necroptosis does not always require RIPK1 function (20). A differential damage-dependent requirement of RIPK1 and RIPK3 for induction of liver tissue damage was also supported by another study (21), indicating that, in addition to 
A

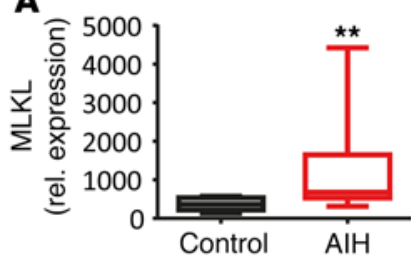

B

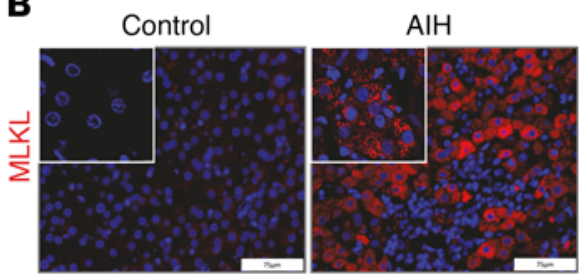

C
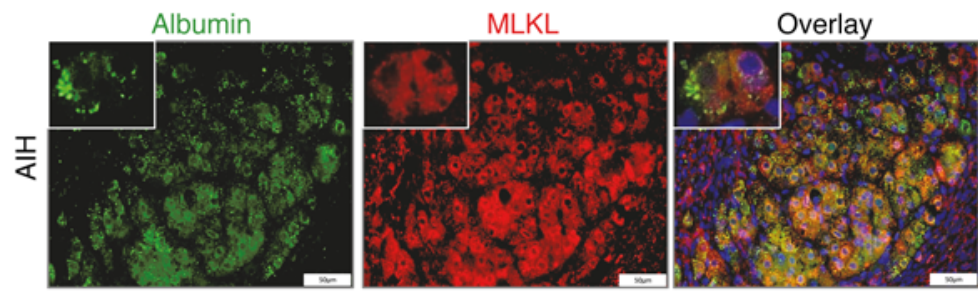

D

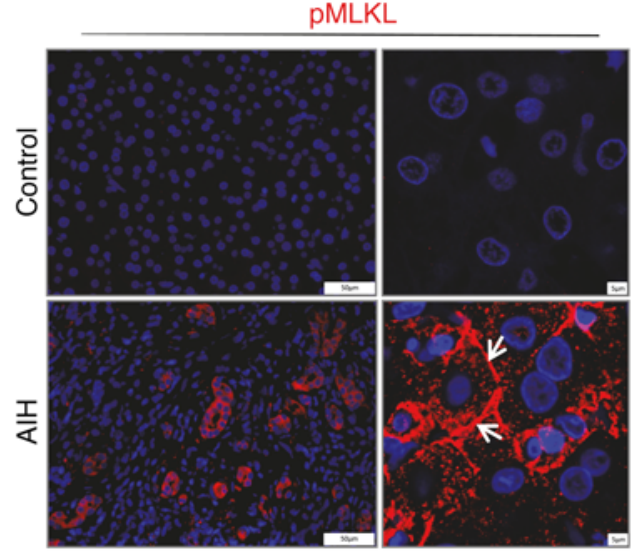

E

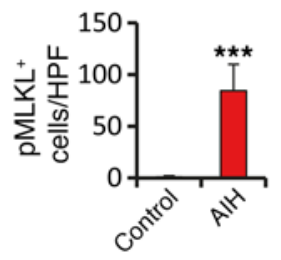

Figure 1. MLKL is upregulated in areas of severe inflammation and cell death during AIH. (A) Quantification of hepatic $M L K L$ mRNA in liver biopsies obtained from control $(n=10)$ or AlH patients $(n=10) .{ }^{* *} P<0.01$ by paired Student's $t$ test. (B) Representative images of liver tissue paraffin sections analyzed for the presence of MLKL by immunofluorescence staining. (C) Representative images showing double immunofluorescence staining of MLKL and albumin in tissue paraffin sections of AlH liver. Inset magnification: $\times 3.38$ (D) Representative images of pMLKLstained sections of control or AlH liver (arrows demonstrate subcellular MLKL localization to plasma membranes; images on the right are confocal images) and (E) quantification of pMLKL-positive hepatocytes ( $n=10$ per group). ${ }^{* *} P<0.001$ by paired Student's $t$ test. Error bars indicate $\pm \mathrm{SD}$, gene expression levels are shown relative to HPRT. Scale bar: $5 \mu \mathrm{m}$ (D, right); $50 \mu \mathrm{m}$ (C and D, left); $75 \mu \mathrm{m}$ (B); $2.77 \mu \mathrm{m}$ (B, inset) canonical necroptosis, other related pathways of programmed necrosis may contribute to hepatocellular death. In particular, the significance of MLKL, the so-far most-terminal known end-stage effector of the necroptosis pathway, in the context of liver injury remains unclear at present.

Autoimmune hepatitis (AIH) is a severe disease associated with chronic inflammation and fibrotic reorganization of liver tissue. The pathology of AIH is characterized by progressive destruction of the hepatic parenchyma due to incompletely understood immune mechanisms that include activation of components of both the innate and the adaptive immune system (22). It has been shown that the severity of AIH correlated with the hepatic presence of immune cells that stain positive for the proinflammatory cytokines IFN- $\gamma$ and TNF- $\alpha$ (23). Moreover, several compelling findings in mouse models clearly demonstrated that the presence and upregulation of these cytokines directly drives hepatocellular cytotoxicity (24). Liver-resident or recruited T lymphocytes, NKT cells, and NK cells have been identified as primary producers of IFN- $\gamma$ during hepatic inflammation, and IFN- $\gamma$ signaling provokes death of hepatocytes through mechanisms that are poorly understood and likely involve several signaling pathways (25). IFN- $\gamma$ rapidly stimulates STAT1 phosphorylation in vivo and in primary hepatocytes (26), and the IFN- $\gamma /$ STAT1 axis has been suggested to block hepatocyte proliferation and induce hepatocyte death via apoptosis (27). However, to what extent IFN- $\gamma$-induced apoptosis contributes to death of hepatocytes in vivo remains unclear, and a detailed understanding of the complex roles of IFN- $\gamma$ in the development of organ-specific autoimmunity is still lacking.

Here, we demonstrate that the pseudokinase MLKL, a key factor in the cellular necrosome, is upregulated and activated in human AIH and in a model of inflammation-dependent hepatitis. Using MLKL-deficient ( $\left.M l \mathrm{kl}^{--}\right)$mice, we have identified MLKL as a key trigger of experimental hepatitis in vivo. Surprisingly, we found that hepatocellular necrosis in experimental hepatitis is driven by a previously unrecognized RIPK3-independent function of MLKL. Moreover, we provide compelling evidence that the wellknown cytotoxic activity of the proinflammatory cytokine IFN- $\gamma$ in hepatic inflammation is strongly connected to induction of MLKL expression via activation of the transcription factor STAT1.

\section{Results}

$M L K L$ is strongly upregulated in hepatocytes of AIH patients and in experimental hepatitis. Although necrosis and necroinflammation are central features of many liver diseases, the role of programmed necrosis in the context of inflammation-dependent hepatocellular death remains to be fully determined. Given that MLKL has been identified as a key mediator and potential biomarker of regulated necrosis $(7,16)$, we initially determined hepatic expression of MLKL in patient cohorts with diverse liver diseases of viral, toxic, or autoimmune origin. While the abundance of $M L K L$ transcripts was very low in healthy controls, patients with steatosis, and patients with primary biliary cirrhosis, we observed highly 
A

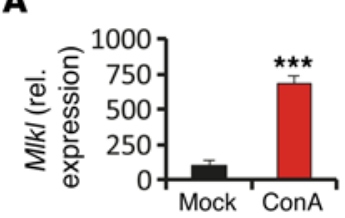

D
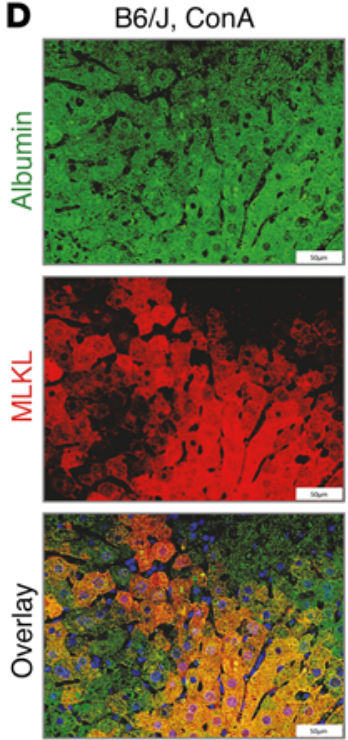

B

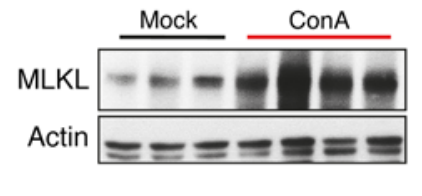

E
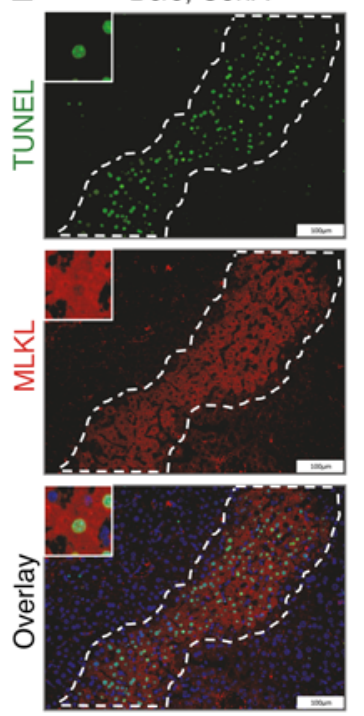

C

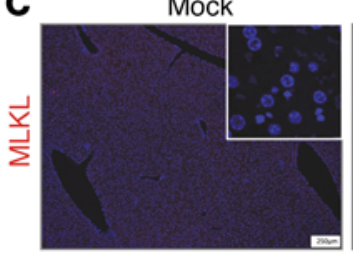

$\mathbf{F}$
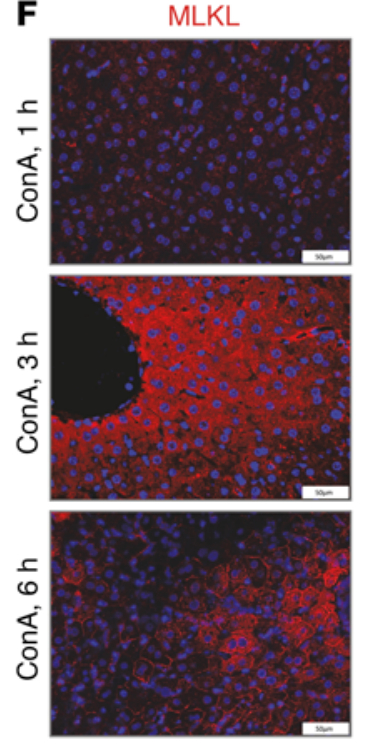

ConA
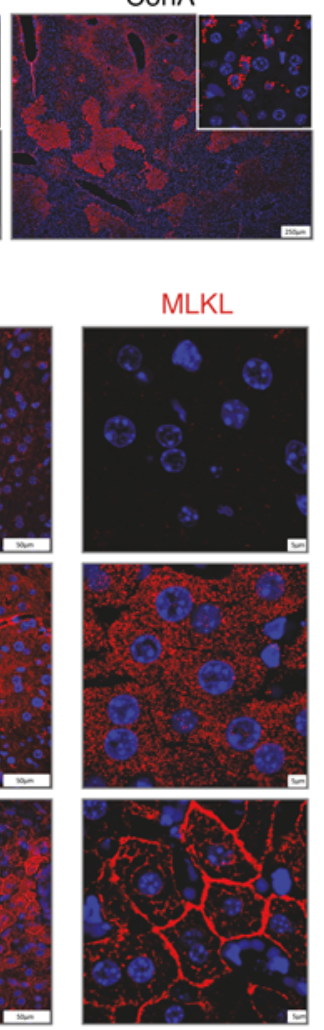

Figure 2. MLKL is strongly upregulated in hepatocytes in experimentally induced hepatitis. C57BL/6 mice were subjected to ConA or saline treatment (mock) as described in the Methods and (A-E) analyzed 7 hours later or $(\mathbf{F})$ at different time points after administration. Experiments were repeated 3 times with similar results. (A) Quantification of hepatic MIkI mRNA by qPCR ( $n>3$ per group). ${ }^{* *} P<0.001$ by paired Student's $t$ test. (B) Detection of MLKL protein in tissue lysates by Western blot. Actin was used as loading control. (C) Representative images of liver tissue paraffin sections analyzed for the presence of MLKL by immunofluorescence staining. Insets show confocal images. (D and E) Representative images showing double staining for (D) MLKL and albumin or (E) MLKL and TUNEL in liver tissue sections (the dashed lines separate necrotic areas from nonnecrotic areas). (F) Representative MLKL staining in liver tissue of wild-type mice challenged at the indicated time points with ConA. Images on the right are confocal images. Error bars indicate $+\mathrm{SD}$, gene expression levels are shown relative to Hprt. Scale bar: $5 \mu \mathrm{m}$ (F, right); $50 \mu \mathrm{m}$ (D and F, left); $100 \mu \mathrm{m}$ (E); $250 \mu \mathrm{m}$ (C); $1.25 \mu \mathrm{m}$ (C) inset). Magnification in (E): $\times 3.38$.

increased hepatic $M L K L$ expression in patients with AIH (Figure 1A and Supplemental Figure 1A; supplemental material available online with this article; doi:10.1172/JCI87545DS1). Moreover, we also observed strong MLKL immunostaining in biopsies obtained from AIH patients. Remarkably, MLKL immunostaining in these samples was particularly restricted to hepatocytes in areas of severe inflammation and hepatocellular death (Figure 1, B and C). Phosphorylation of MLKL (pMLKL) and translocation of pMLKL to the plasma membrane has been demonstrated to be essential for necroptotic cell death $(10,28)$. Accordingly, strong pMLKL signals were present in affected areas in AIH tissue, whereas no pMLKL immunostaining was identified in healthy control tissues (Figure 1, D and E). Moreover, confocal microscopy clearly revealed subcellular MLKL and pMLKL localization to plasma membranes (Figure 1D and Supplemental Figure 1B), suggesting that MLKL activation is linked to disease activity in AIH.

In order to study a potential role for MLKL in liver inflammation, we next took advantage of a mouse model in which hepatitis is induced by intravenous injection of the lectin concanavalin A (ConA), which resembles some aspects of immunemediated hepatitis in humans $(29,30)$. Whereas MLKL expression in steady-state murine liver tissue was weak, treatment of mice with ConA resulted in a rapid and strong increase in both $\mathrm{Mlkl}$ transcripts and protein, as evidenced by qPCR (Figure 2A and Supplemental Figure 2A), Western blot analysis (Figure 2B and Supplemental Figure 2B), and immunohistochemistry (Figure 2C and Supplemental Figure 2F). The absence of specific signals in $\mathrm{Mlkl}^{-/}$mice confirmed the specificity and suitability of the antibody used for detection (Supplemental Figure 2C). Immunofluorescent microscopy further localized inflammation-dependent MLKL accumulation to hepatocytes, as demonstrated by double staining for MLKL and the hepatocyte marker albumin (Figure 2D and Supplemental Figure 2D). Moreover, expression of $M l k l$ was pronounced in necrotic areas stained positive for the cell death marker TUNEL (Figure 2E). Accordingly, we observed that hepatic $M l k l$ mRNA expression correlated well with concentrations of circulating transaminases (Supplemental Figure 2E). Notably, at 3 hours after ConA injection, the MLKL protein was equally distributed throughout the cytoplasm of hepatocytes (Figure 2F). By contrast and similar to the staining pattern we observed in human $\mathrm{AIH}$, at later stages, when hepatocytes stained positive for TUNEL, MLKL immunostaining was strongly localized to membranous compartments, including 

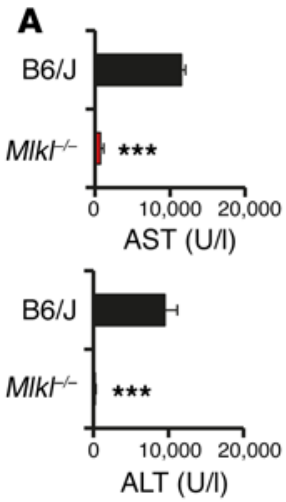

F

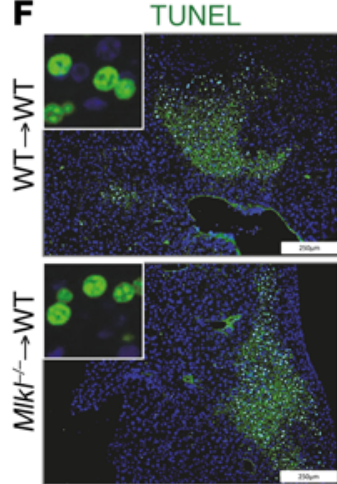

B

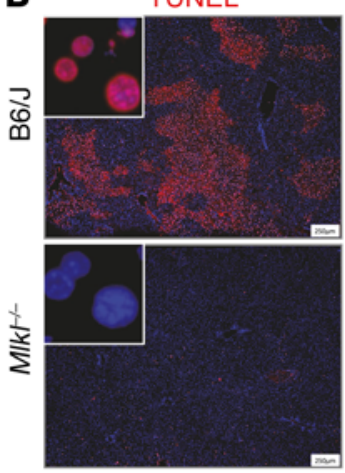

C

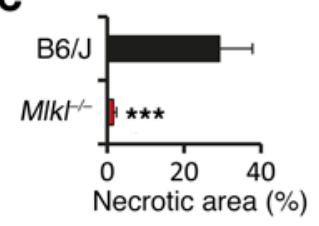

D

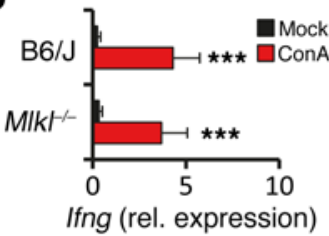

E
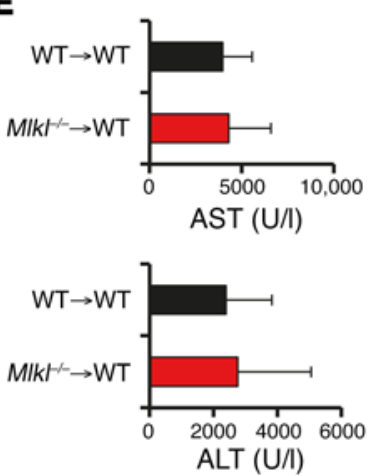

$\mathbf{G}$
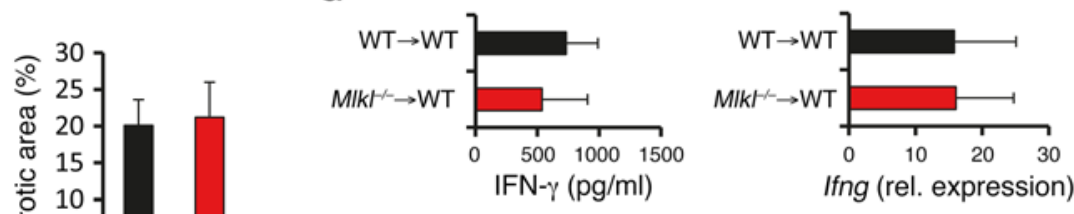

H

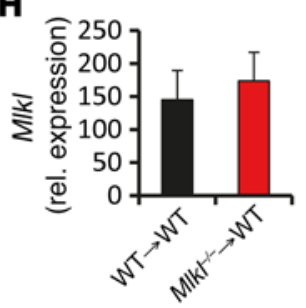

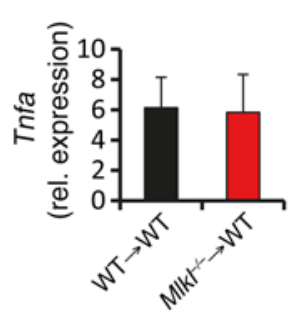

I

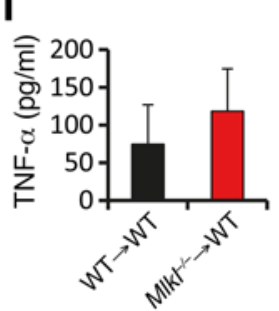

Figure 3. MLKL expression in hepatocytes drives ConA-induced necrotic cell death. (A-D) C57BL/6 and MIK/ ${ }^{-1-}$ mice were i.v. injected with ConA and analyzed 7 hours later. Experiments were repeated 3 times with similar results. (A) Plasma AST/ALT concentrations $\left(n=4\right.$ per group). ${ }^{* * *} P<0.001$ by paired Student's $t$ test. (B) Representative images of liver sections stained by TUNEL assay. (C) Quantification of necrotic areas in TUNEL assay-stained livers of ConA-challenged mice ( $n=4$ per group). ${ }^{* *} P<0.001$ by paired Student's $t$ test. (D) Quantification of Ifng transcripts in liver lysates of mock- or ConA-challenged control and MIkl-- mice by qPCR ( $n>3$ per group). ${ }^{* *} P<0.001$ by paired Student's $t$ test. (E-I) $1 \times 10^{7}$ bone marrow cells isolated from control or $\mathrm{Mlkl}^{-1-}$ mice were i.v. injected into lethally irradiated C57BL/6 recipient mice. Eight weeks later, mice were injected with ConA ( $n=5$ per group). (E) Plasma AST/ALT concentrations ( $n=5$ per group). (F) Representative images and quantification ( $n>4$ per group) of TUNEL assay-stained tissue sections. (G) Quantification of serum IFN- $\gamma$ concentrations and hepatic Ifng transcripts in ConA-treated animals ( $n=5$ per group). (H) Quantification of hepatic MIkI and Tnfa transcripts ( $n=5$ per group). (I) Quantification of TNF- $\alpha$ in supernatants of splenocytes isolated from ConA-challenged mice and stimulated ex situ with PMA/ionomycin for 24 hours ( $n=5$ per group). Error bars indicate + SD, gene expression levels are shown relative to Hprt. Scale bar: $250 \mu \mathrm{m}$. Original magnification in $(\mathbf{B}$ and $\mathbf{F}): \times 7.55$.

the plasma membrane, the nuclear membrane, and membranous compartments with morphological features of mitochondria (Figure $2 \mathrm{~F}$ and Supplemental Figure 2, F and G). As it was previously shown that phosphorylation of MLKL coincides with MLKL oligomerization and membrane translocation, these data strongly implicate MLKL activation in hepatocellular necrosis in the context of human and mouse inflammation-dependent liver disease.

Hepatocellular MLKL expression drives ConA-induced hepatitis. In order to provide direct functional evidence for a role of the MLKL protein in experimental hepatitis, we subjected $\mathrm{Mlkl}^{-/-}$mice to ConA treatment. Interestingly, $\mathrm{Mlkl}^{\mathrm{H}^{--}}$animals had largely diminished plasma aspartate aminotransferase (AST) and alanine aminotransferase (ALT) concentrations (Figure 3A), compared with those in wild-type controls. Moreover, TUNEL staining revealed significantly reduced liver tissue necrosis and death of hepatocytes in $\mathrm{Mlkl}^{-/}$mice (Figure 3, B and C). Our data so far clearly point to a hepatocyte-intrinsic role of MLKL during inflammation-dependent cell death. Consistently, IFN- $\gamma$ serum concentrations and production by immune cells, which has been shown to be a central trigger for hepatocellular death in the ConA model, were not significantly altered in $\mathrm{Mlkl}^{-/-}$mice (Figure 3D and Supplemental Figure 3A). We have previously shown that STAT1 activation triggers IFN- $\gamma$-dependent hepatocellular death (26). In order to investigate whether MLKL deficiency affects hepatic STAT1 signaling, we compared STAT1 activation in ConA-challenged wild-type and $\mathrm{Mlkl}^{-/-}$mice. While hepatic pSTAT $1^{\mathrm{Tyr} 701}$ was not detectable in unchallenged animals, ConA administration induced a marked induction of PSTAT1 ${ }^{\mathrm{Tyr} 701}$ in livers of control and $M l k l^{--}$mice (Supplemental Figure 3B). In addition, primary mouse hepatocytes (PMHs) stimulated ex situ with sera of ConA-challenged wild-type or $\mathrm{Mlkl}^{-/}$mice released similar amounts of the cytotoxicity marker LDH (Supplemental Figure $3 \mathrm{C}$ ), suggesting that proinflammatory cytokine responses in $\mathrm{Mlkl}^{-/-}$mice are not reduced to an extent that they substantive- 
A

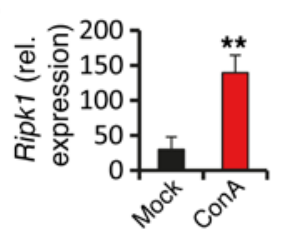

C

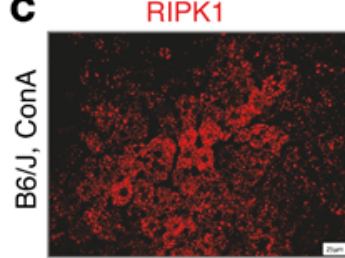

RIPK1

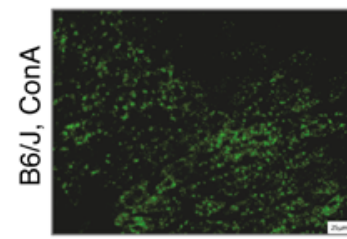

D

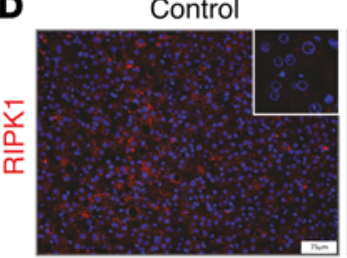

B

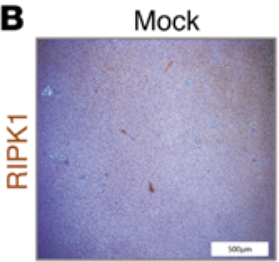

Albumin

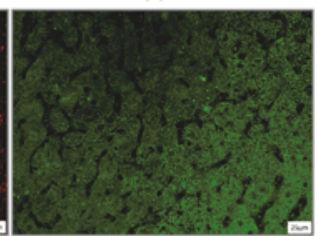

TUNEL

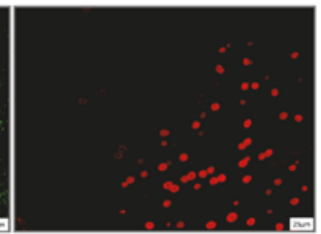

AlH

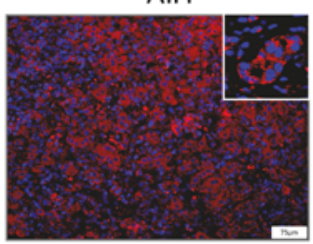

ConA
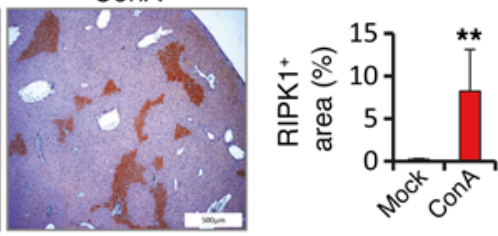

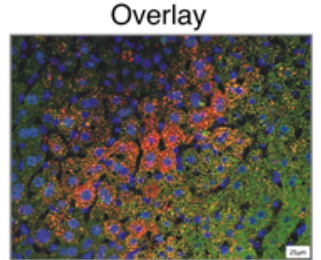

Overlay

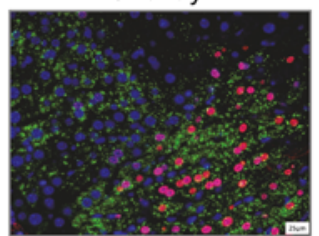

E

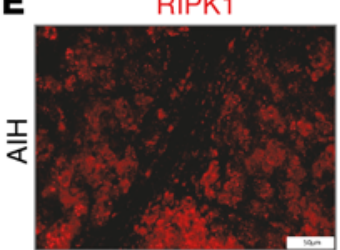

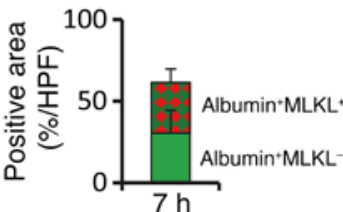

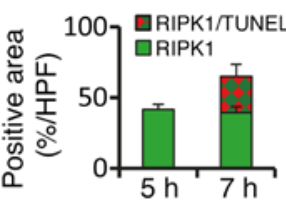

Albumin/RIPK1

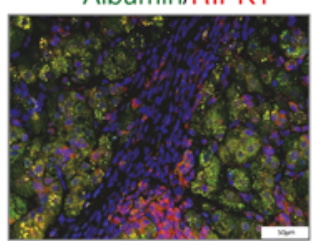

Figure 4. RIPK1 is upregulated during experimentally induced hepatitis and in human AlH. (A-C) C57BL/6 mice were i.v. injected with ConA and analyzed 7 hours later. Experiments were repeated 3 times with similar results. (A) Hepatic Ripk1 transcripts were quantified by qPCR ( $n>3$ per group). ${ }^{* *} P<0.01$ by paired Student's $t$ test. (B) Representative images of liver tissue paraffin sections analyzed for the presence of RIPK1 by immunohistochemistry and quantification of RIPK1-positive areas in immunohistochemistry ( $n>4$ per group). ${ }^{* *} P<0.01$ by paired Student's $t$ test. (C) Representative images and quantification of double staining of livers sections for RIPK1 and albumin and TUNEL assay. (D and E) Representative images of liver samples of AlH patients stained for (D) RIPK1 alone or for (E) RIPK1 and albumin. Error bars indicate +SD, gene expression levels are shown relative to Hprt. Scale bar: $25 \mu \mathrm{m}$ (C); $50 \mu \mathrm{m}$ (E); $75 \mu \mathrm{m}$ (D); $1.54 \mu \mathrm{m}$ (D, inset); $500 \mu \mathrm{m}$ (B).

ly contribute to the protected phenotype of these mice in ConA hepatitis. Although it was previously shown that $\mathrm{Mlkl}^{-/-}$mice did not display a hematological disturbance under steady-state conditions (31), we further addressed the role of MLKL in hematopoietic cells in chimeric mice generated by adoptive transfer of

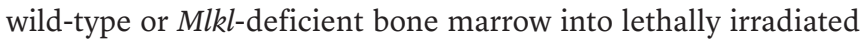
wild-type recipient mice. Subsequently, administration of ConA induced a similar degree of liver injury in both chimeric strains, as AST and ALT plasma concentrations and TUNEL positivity of hepatocytes were comparable in both groups (Figure 3, E and F). Accordingly, in both chimeric strains, hepatic Mlkl, Ifng, and Tnfa transcripts; serum concentrations of IFN- $\gamma$; and production of TNF- $\alpha$ by splenocytes were similarly increased (Figure 3, G-I). These data suggest that hepatocyte-intrinsic MLKL expression rather than functions in hematopoietic cells are required to mediate ConA-induced liver injury.

Recently, increased pMLKL staining was described around central veins in areas with significant damage in patients suffering from DILI (16). Therefore, we elucidated the potential contribution of MLKL to murine DILI by measurement of hepatic $M l k l$ expression in livers of mice subjected to APAP overdose. Howev- er, in contrast to that in the ConA model, hepatic $M l k l$ transcripts were not increased and were comparable to saline-treated animals (Supplemental Figure 3D), suggesting that MLKL activation is not a pivotal cell death trigger in response to APAP. In line with this and previous reports (19), $M l k l^{--}$mice developed strong APAPdependent liver injury, as demonstrated by plasma AST and ALT determination (Supplemental Figure 3E), H\&E and TUNEL staining, and quantification of the necrotic areas (Supplemental Figure 3F). Similarly, no changes in the cell death response were evident in PMHs that were isolated from wild-type and $\mathrm{Mlkl}^{-/-}$mice and treated ex situ with APAP (Supplemental Figure 3G). Collectively, our data demonstrate that MLKL is essential for inflammationinduced necrotic hepatocellular death.

RIPK1 but not RIPK3 activity is required for induction of experimental hepatitis. RIPK1 is known to regulate the RIPK3-dependent phosphorylation and activation of MLKL, which is a hallmark of the canonical necroptosis pathway $(7,31-37)$. However, the role of these RIP kinases in models of experimental induced liver injury remains controversial and incompletely understood. Therefore, we next aimed to characterize the role of these RIPK proteins for the execution of MLKL-dependent hepatocellular death in the 

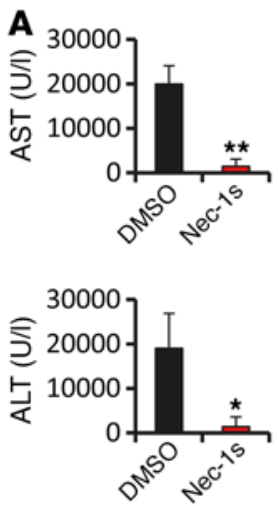

B
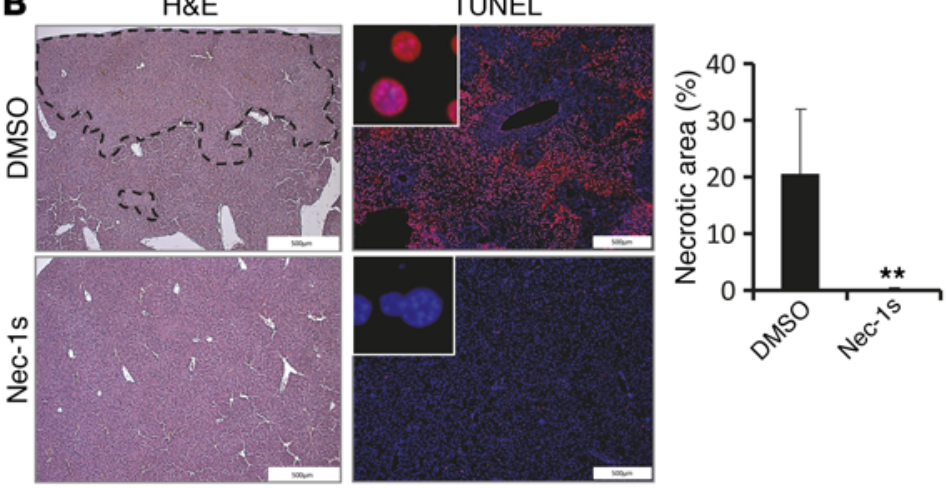

C

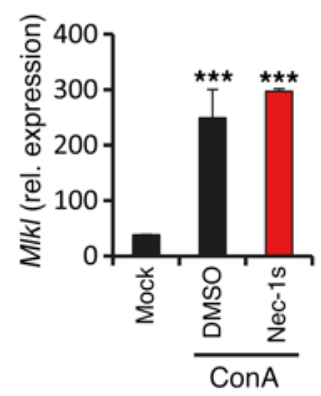

D

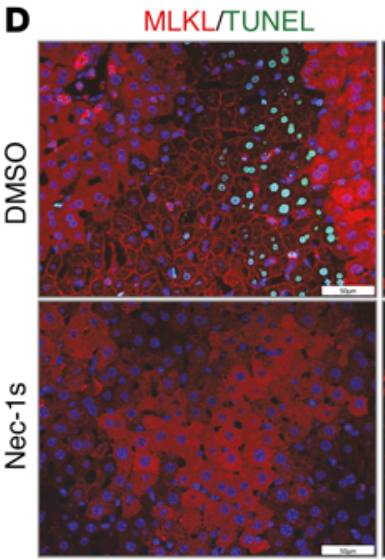

MLKL

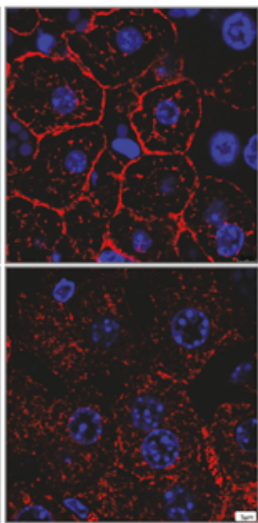

Figure 5. ConA-induced necrotic cell death depends on the kinase activity of RIPK1. C57BL/6 mice were treated with vehicle (DMSO) or nec-1s 30 minutes prior to ConA administration. Experiments were repeated 3 times with similar results. (A) Plasma concentrations of ALT and AST ( $n=4$ per group). ${ }^{*} P<0.05,{ }^{* *} P<0.01$ by paired Student's $t$ test. (B) Representative images of H\&E- (dashed lines represent necrotic areas) and TUNEL assay-stained liver tissue sections and quantification of necrotic area ( $n=4$ per group). ${ }^{*} P<0.01$ by paired Student's $t$ test. (C) Mlkl transcripts in unchallenged mice (mock) and mice treated with ConA alone (DMSO) or pretreated with nec-1s (nec-1s) were quantified by qPCR ( $n>3$ per group). ${ }^{* * *} P<0.001$ by paired Student's $t$ test. (D) Representative images of liver tissue sections from ConA-treated mice stained for MLKL in combination with TUNEL assay. Images on the right are confocal images (MLKL staining alone). Error bars indicate $+\mathrm{SD}$, gene expression levels are shown relative to Hprt. Scale bar: $5 \mu \mathrm{m}$ (D, right); $50 \mu \mathrm{m}$ (D, left); $500 \mu \mathrm{m}$ (B); $\times 7.55$ (B, insets).
ConA model. Similar to MLKL, RIPK1 was profoundly and rapidly upregulated at both the mRNA and the protein level in livers after ConA treatment (Figure 4, A and B, and Supplemental Figure 4, A and B). Immunohistochemistry further revealed that RIPK1 staining clustered around hepatocytes in large foci at areas of pronounced tissue necrosis (Figure 4C and Supplemental Figure 4, $\mathrm{B}$ and $\mathrm{C})$. In analogy to these findings in mice, we also observed a clustered RIPK1 staining pattern in hepatocytes in human AIH, whereas RIPK1 was only weakly expressed in the healthy liver (Figure 4, D and E). To ascertain whether ConA-induced liver injury depends on the kinase activity of RIPK1, we treated wildtype mice with the RIP1 kinase inhibitor necrostatin-1s (nec-1s) prior to ConA injection. Indeed, we observed low serum transaminase concentrations (Figure 5A) and reduced necrosis (Figure 5B) in mice that received additionally nec-1s. Importantly, inhibition of RIPK1 kinase activity by nec-1s blocked the translocation of MLKL to the plasma membrane, while the induction of $M l k l$ gene expression in response to ConA was not affected (Figure 5, C and D). Notably, pretreatment with nec-1s had no impact on hepatic expression of Ifng, suggesting that the kinase activity of RIPK1 is involved in ConA-induced hepatocellular death downstream of $\mathrm{T}$ cell activation (Supplemental Figure 4D). In summary, these data indicate that RIPK1 is involved in MLKL-mediated necrotic damage during hepatic inflammation.

Although we observed only weak expression of RIPK3 in healthy murine liver tissue as compared with other organs (Supplemental Figure 5, A and C), RIPK3 has been reported to be involved in the development of liver damage induced by APAP or alcohol overdose $(17,20)$. However, in contrast to Ripk1 and Mlkl, Ripk3 hepatic transcripts were not increased in response to ConA or in human AIH (Supplemental Figure 5B). In line with these findings, we were unable to detect RIPK3 in hepatocytes by immunohistochemistry, and even late necrotic hepatocytes, in which the breakdown of plasma membranes is traceable by $\beta$-catenin staining, did not stain positive for RIPK3 (Figure 6A). By contrast, we observed RIPK3 protein positivity in stromal cells that primarily represent liver-resident F4/80 ${ }^{+}$Kupffer cells (Figure 6A and Supplemental Figure 5E). The absence of specific signals in Ripk3 $3^{-1}$ mice confirmed the specificity and suitability of the antibody used for detection (Supplemental Figure 5, C and D). Consistently, Ripk3 transcription was low in cultured PMHs (Supplemental Figure 5A). To study the functional contribution of RIPK3 to ConA-induced hepatocellular death, we next subjected Ripk3/- mice to ConA treatment. In sharp contrast to the protection of mice in the context of RIPK1 inhibition and MLKL deficiency, Ripk3 ${ }^{--}$mice had high plasma aminotransferase concentrations and developed a similar degree of histological liver damage as wild-type control mice (Figure 6, B and C, and Supplemental Figure 5, F and G). In addition, TUNEL staining and quantification of TUNEL-positive cells identified no changes in the degree of hepatocellular necrosis in livers of Ripk3 $3^{--}$mice (Figure 6, B and C). To further evaluate these unexpected findings, we analyzed the subcellular localization of MLKL in hepatocytes of ConA-injected Ripk3 $3^{-/}$mice by confocal microscopy and Western blotting. Interestingly, similar to observations in wild-type mice, the MLKL protein clearly accumulated in affected areas of the plasma membrane in Ripk3 ${ }^{-1}$ 
A $\beta$-Catenin/RIPK3

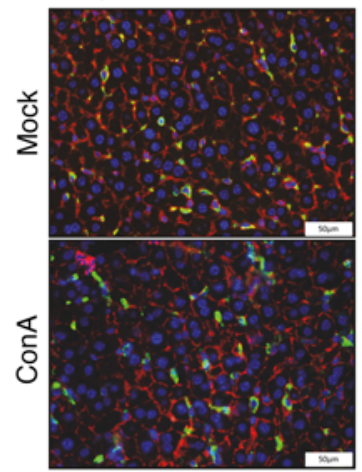

D

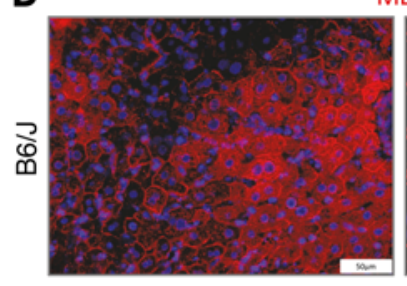

$\beta$-Catenin/RIPK3

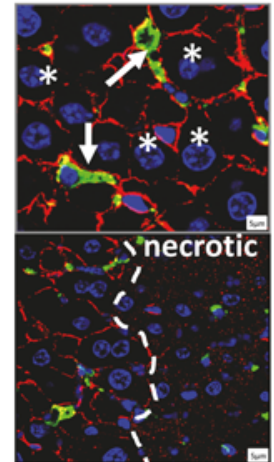

MLKL

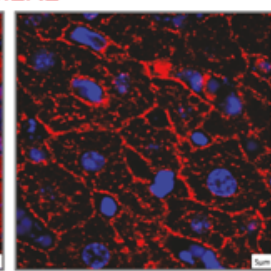

B
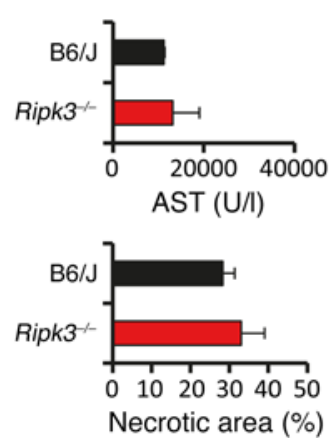

C TUNEL

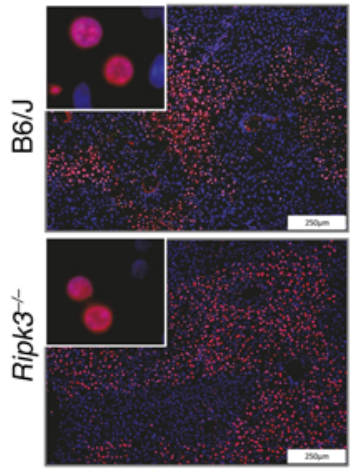

MLKL

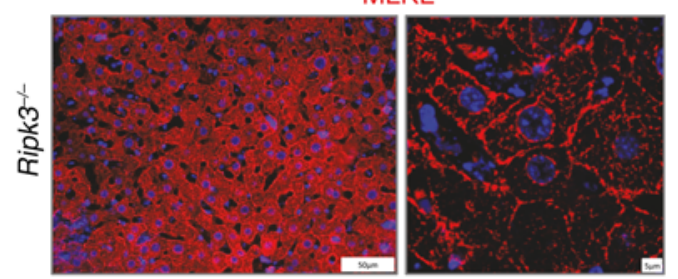

$\mathbf{F}$

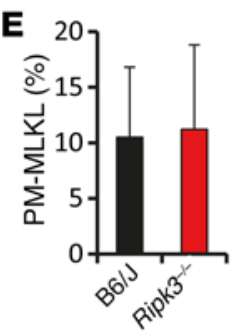

Na,K-ATPase

GAPDH

$$
\frac{\text { Ripk3 }^{-}, \text {ConA }}{\text { C PM C PM }}
$$

G

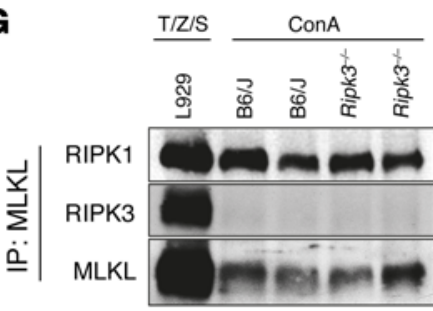

H

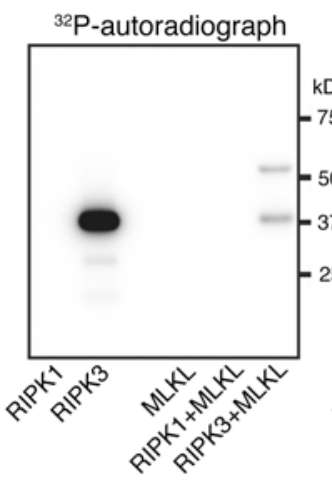

Coomassie

$<B S A$

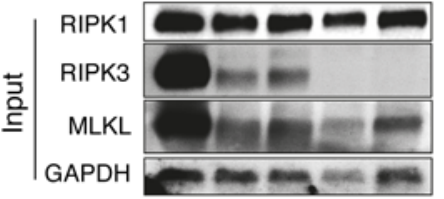

Figure 6. RIPK3 is dispensable for ConA-induced hepatic injury. C57BL/6 and Ripk3-/- mice were i.v. injected with ConA and analyzed 7 hours later. Experiments were repeated 3 times with similar results. (A) Representative images of liver tissue sections double stained for RIPK3 and $\beta$-catenin (confocal images are on the right; the dashed line separates necrotic areas from nonnecrotic areas; arrows demonstrate RIPK3-positive immune cells; asterisks mark representative hepatocytes). (B) Plasma AST concentrations of ConA-challenged control and Ripk3 $3^{-/-}$mice ( $n>3$ per group) and evaluation of necrotic areas in TUNEL assay-stained liver sections of control and Ripk $3^{-/-}$mice (from C, $n>3$ per group). (C) Representative images of immunohistochemical (TUNEL assay) staining analysis of hepatic tissue sections. (D) Representative images of MLKL-stained liver tissue sections. (E) Quantification of plasma membrane localized MLKL (from $\mathbf{D}, n>4$ per group). (F) Western Blot analysis demonstrating that endogenous MLKL locates at the plasma membrane (PM) in Ripk3 ${ }^{-/-}$mice following ConA treatment. C; cytoplasm. (C) Endogenous MLKL was immunoprecipitated with anti-MLKL antibody in lysates of L929 cells (treated for 3 hours with TNF- $\alpha / z$ VAD/SMAC mimetic) or in liver lysates of ConA-challenged wild-type or Ripk3 $3^{-/-}$mice. (H) Recombinant hRIPK3, but not hRIPK1, kinase domain undergoes autophosphorylation and can mediate hMLKL phosphorylation in vitro. Phosphoryl transfer of ${ }^{32} \mathrm{P}$ by $25 \mathrm{ng} / \mu \mathrm{l}$ hRIPK1 or hRIPK3 kinase domain in the absence of hMLKL, or $75 \mathrm{ng} / \mu \mathrm{hMLKL}$ in the absence of kinase, or $2.5 \mathrm{ng} / \mu \mathrm{hRIPK} 1$ or hRIPK3 in the presence of $75 \mathrm{ng} / \mu \mathrm{hMLKL}$ was detected by autoradiography. A Coomassie-stained image of the autoradiograph is shown. The images are representative of triplicate experiments. Error bars indicate $+\mathrm{SD}$, gene expression levels are shown relative to HPRT/Hprt. Scale bar: $5 \mu \mathrm{m}(\mathbf{A}$, right, and $\mathbf{D}$, right); $50 \mu \mathrm{m}(\mathbf{A}$, left, and $\mathbf{D}$, left); $250 \mu \mathrm{m}$ (C); magnification in (C); $\times 11$. 
A

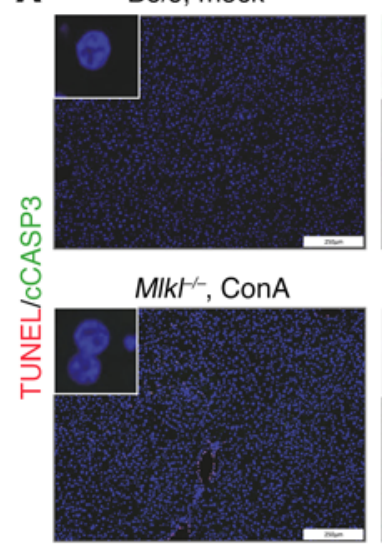

C
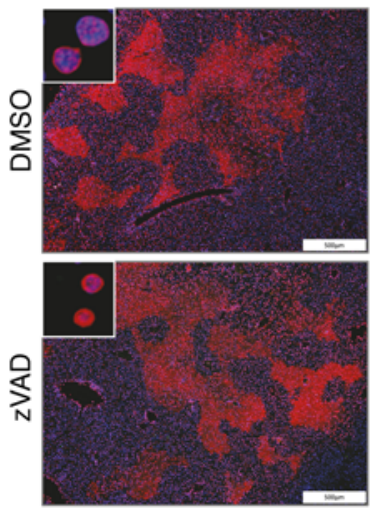

B6/J, GalN/LPS

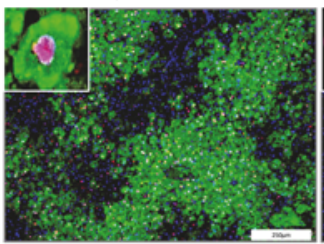

Ripk3־, ConA
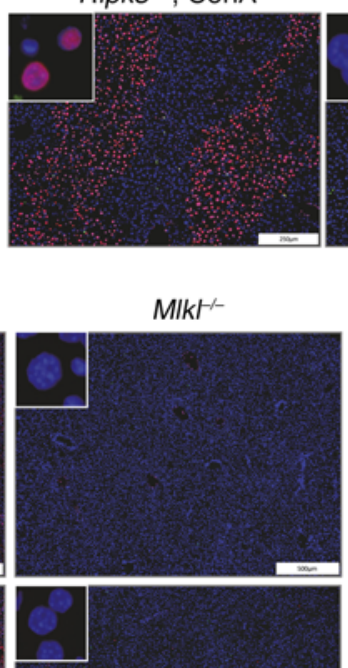

B6/J, ConA

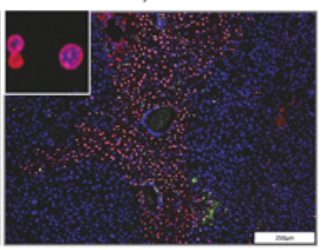

$B 6 / J+$ nec-1s, ConA

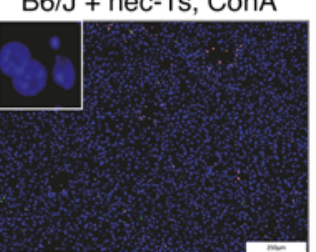

B
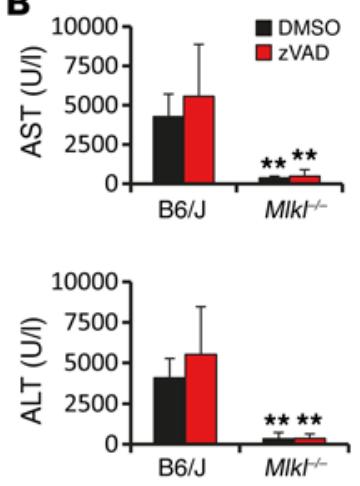

Figure 7. Caspases are not required for ConA-induced hepatocellular death. (A) Representative images showing double immunofluorescence staining of cleaved caspase-3 and TUNEL assay in liver paraffin sections of unchallenged (mock), LPS/-GalN, or ConA-treated mice. (B and C) C57BL/6 or M/kl/- mice were treated with vehicle (DMSO) or ZVAD 15 minutes prior to ConA administration. (B) Plasma AST/ALT concentrations ( $n>3$ per group). ${ }^{* *} P<0.01$ by paired Student's $t$ test. (C) Representative images of TUNEL assay-stained liver sections and quantification of necrotic area in liver cross sections of ConA-challenged mice ( $n>3$ per group). ${ }^{* *} P<0.001$ by paired Student's $t$ test. Error bars indicate + SD. Scale bar: $250 \mu \mathrm{m}$, magnification $\times 8.25$ (A); $500 \mu \mathrm{m}$, magnification $\times 8.65$ (C).

mice, as demonstrated by immunohistochemical analysis and Western blotting (Figure 6, D-F, and Supplemental Figure 5H), suggesting that hepatocellular MLKL activation in ConA hepatitis does not require RIPK3. Moreover, similar to ConA-challenged wild-type animals, hepatocellular necrosis was accompanied by profound clustering of RIPK1 into large foci in hepatocytes of Ripk3 $^{-/}$mice (Supplemental Figure 5I). During canonical TNF- $\alpha-$ mediated necroptosis, MLKL interacts with RIPK3 through its kinase-like domain within the necrosome complex (7). In order to test whether MLKL interacts with RIP kinases in the context of inflammation-dependent programmed hepatocellular necrosis, we next immunoprecipitated endogenous MLKL in liver lysates of ConA-challenged wild-type and Ripk3-/- animals. Treatment of L929 cells with TNF- $\alpha$ plus zVAD and SMAC mimetic served as a positive control for transient interaction of MLKL with RIPK3. Interestingly, we observed that MLKL is part of a complex that contains RIPK1 but not RIPK3 in the setting of ConA hepatitis, suggesting that MLKL- and RIPK1-containing protein complexes may assemble in the absence of RIPK3 (Figure 6G).

Collectively, these data clearly indicate that RIPK3 but not RIPK1 is dispensable for ConA-induced hepatocellular necrosis.
Thus, in the setting of inflammation-dependent liver disease, a noncanonical type of regulated necrosis that does not require MLKL phosphorylation by RIPK3 is a critical trigger of hepatocellular death. Notably, RIPK1 itself was not able to directly phosphorylate MLKL in in vitro kinase assays, suggesting that additional mechanisms beyond RIPK3-mediated phosphorylation exist, where as-yet unidentified kinases could mediate MLKL activation (Figure 6H).

$M L K L$ is not required for LPS/GalN-dependent hepatocellular apoptosis. While tissue necrosis has been clearly implicated in ConA-induced liver inflammation, the role of hepatocellular apoptosis in this model remains controversial. In comparison to mice challenged with LPS/D-galactosamine (LPS/GalN) (Figure 7A and Supplemental Figure 6A), a model known to be primarily mediated by apoptotic hepatocellular death, we observed only weak activation of caspase-3 in liver areas with strong hepatocellular death in $\mathrm{C} 57 \mathrm{BL} / 6$ wild-type mice subjected to ConA treatment (Figure 7A and Supplemental Figure 6A). Similarly, no cleaved caspase-3 signals were present in ConA-treated $\mathrm{Mlkl}^{-/-}$or $\mathrm{Ripk3}^{-/-}$mice and mice pretreated with nec-1s (Figure 7A and Supplemental Figure $6 \mathrm{~A})$. These data suggest that apoptotic cell death does not substantially contribute to cell death during ConA-induced liver injury. In 
A

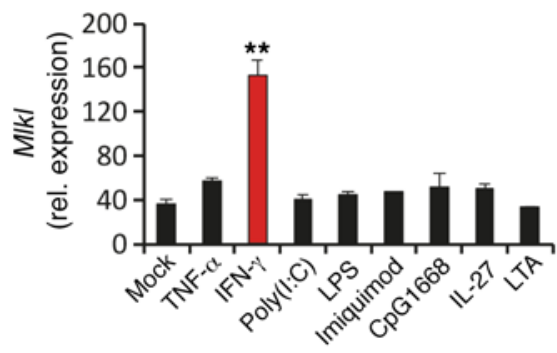

B

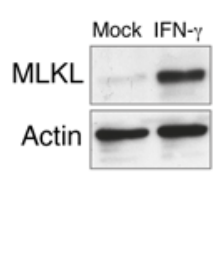

C

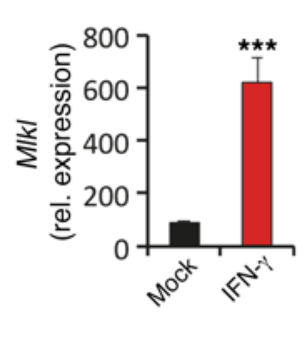

MLKL

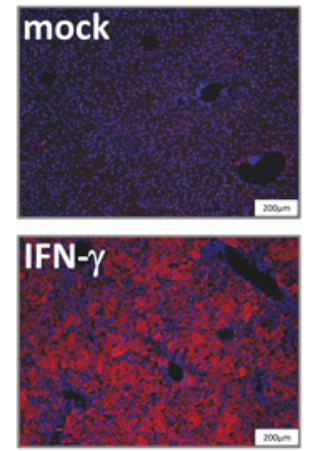

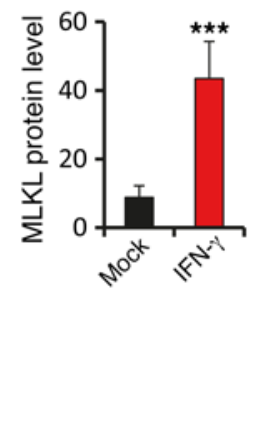

D

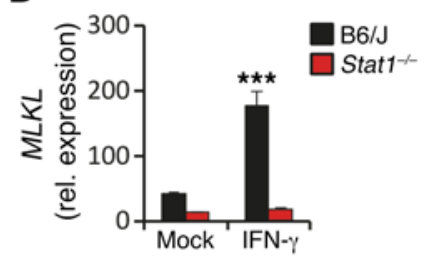

E

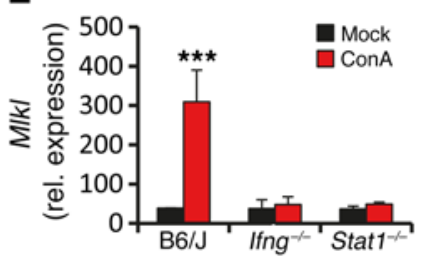

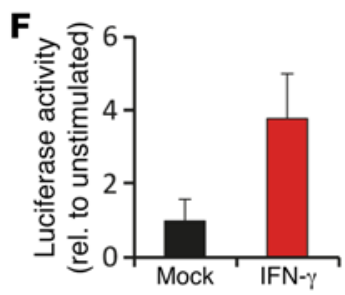

G
H<smiles>C[131I]C</smiles>
ACTGATGATCCTACAGGAACTGAGCGCAGGATAGÁCCGGACGAGCGCCCCTCGGAGATCACGT TGACTACTAGGATGTCCTTGACTCGCGTCCTATCTGGCCTGCTCGCGGGGAGCCTCTAGTGCATCTTAGGTCACTCGACATCGCGCCGTCCTC

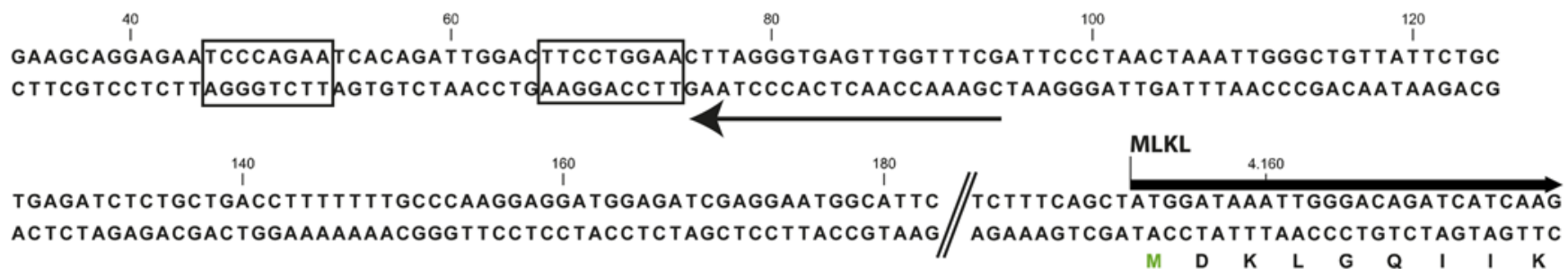

Figure 8. IFN- $\gamma$-dependent STAT1 activation regulates MLKL expression in experimental hepatitis. (A) MIkl transcripts and (B) MLKL protein were quantified in PMHs stimulated ex situ with indicated factors ( $n=3$ per group). (C) IFN- $\gamma$ expression constructs or empty control vectors (mock) were injected into C57BL/6 mice. Four days later, hepatic MIkl mRNA or MLKL protein ( $n=3$ per group) was quantified by qPCR or Western blot. The presence of MLKL protein in livers was determined by specific immunofluorescent staining. (D) Relative MIkI mRNA abundance in primary hepatocytes of wild-type or Stat $1^{-1}$ mice stimulated with IFN- $\gamma$ for 6 hours ( $n=3$ per group). (E) Quantification of hepatic MIkI mRNA in vehicle- (mock) or ConA-challenged control C57BL/6 mice, Ifng ${ }^{-/-}$mice, and Stat1 ${ }^{-/-}$mice ( $n>3$ per group). (F) PMHs were transfected with a Mlkl luciferase promoter construct. Twenty-four hours later, cells were stimulated with IFN- $\gamma$ for 16 hours and firefly luciferase activity was determined (relative to mock group, $n=3$ per group). (C) PMHs were stimulated with IFN- $\gamma$ for 45 minutes. Subsequently ChIP was performed using a control antibody (IgG) or anti STAT1pTyr701 mAbs, as detailed in the Methods. Precipitated DNA fragments were quantified by qPCR using the primer sequences indicated in the sector of Mlkl genomic region in $\mathbf{H}$. Primers covering a previously described STAT1-binding site in the mouse Irf1 gene promoter served as a positive control. All experiments were performed at least 2 times with similar results. (H) Partial sequence of the mouse genomic MIkl region upstream of the start codon. Two areas predicted by Matinspector (60) and MAPPER (61) as putative STAT1-binding sites were highlighted. TSS, predicted transcriptional start site. Error bars indicate + SD, gene expression levels are shown relative to Hprt. Scale bar: $200 \mu \mathrm{m}$ (C). ${ }^{* *} P<0.01,{ }^{* *} P<0.001$ by paired Student's $t$ test. line with this notion, injection of a broad-spectrum caspase inhibitor (zVAD) into control or $\mathrm{Mlkl}^{-/-}$mice before treatment with ConA had no effect on tissue necrosis and the levels of serum transaminases (Figure 7, B and C, and Supplemental Figure 6B).

In order to study a potential role of MLKL for hepatocellular apoptosis, we subjected $M l k l^{--}$mice to LPS/GalN. However, different from the ConA model, $M l \mathrm{kl}^{-/-}$mice developed strong LPS/ GalN-dependent liver injury, as demonstrated by plasma AST and ALT determination and H\&E and cleaved caspase-3 staining (Supplemental Figure 6, C-E), indicating that MLKL deficiency does not attenuate hepatocyte apoptosis in this model.
IFN- $\gamma$-dependent STAT1 activation regulates $M L K L$ expression in experimental hepatitis. Having shown that MLKL is strongly upregulated in experimental hepatitis and $\mathrm{AIH}$, our next objective was to identify potential extracellular activators of $M l k l$ gene transcription. We therefore exposed PMHs ex situ to well-known triggers for necroptosis or cytokines involved in the pathogenesis of inflammatory liver disease. Interestingly, IFN- $\gamma$, a key driver of liver pathology, profoundly increased $M l k l$ transcription (Figure $8 \mathrm{~A}$ ) and protein abundance (Figure $8 \mathrm{~B}$ ). Furthermore, in vivo overexpression of IFN- $\gamma$ by hydrodynamic delivery of DNA minicircles was sufficient to induce strong hepatic $M l k l$ gene expression that 
was restricted to hepatocytes, as demonstrated by MLKL-specific immunostaining (Figure 8C). Given that IFN- $\gamma$ has been shown to primarily mediate its biological function via the transcription factor STAT1, we stimulated primary hepatocytes of control and Stat $^{-1-}$ mice with IFN- $\gamma$ and measured $M l k l$ expression. In support of an IFN- $\gamma /$ STAT1-dependent regulation of $M l k l$ gene expression, IFN- $\gamma$ did not induce $M l k l$ transcription in $S_{t a t 1^{-/}}$mice or STAT1-deficient hepatocytes (Figure 8D and Supplemental Figure 7A). IFN- $\gamma /$ STAT1 signaling is essential for ConA-mediated liver injury, and T cells and NKT cells were identified as primary producers of IFN- $\gamma$ in this model $(26,29)$. Thus, we next analyzed the expression of $\mathrm{Mlkl}$ in ConA-treated $\mathrm{Stat1}^{-/}$, Ifng $\mathrm{fl}^{-/}$, and lymphopenic $\mathrm{Rag1}^{-/-}$mice. Remarkably, in all three strains hepatic $\mathrm{Mlkl}$ expression was not induced in vivo, and this correlated to reduced hepatocyte death and tissue damage (Figure $8 \mathrm{E}$ and Supplemental Figure 7, B-E). Collectively, these data demonstrate that in vivo $M l k l$ expression is induced by an IFN- $\gamma /$ STAT1-dependent pathway requiring the presence of lymphocytes.

Interestingly, in silico analysis identified several potential STAT1-binding sites in the genomic region upstream of the $M l k l$-coding sequence (Figure $8 \mathrm{H}$ ), indicating that $M l k l$ gene transcription may be directly induced by IFN- $\gamma$-dependent STAT1 activation. To address this question, we cloned upstream DNA fragments of the $M l k l$ genomic region and generated luciferase reporter constructs for reporter gene assays. Interestingly, basal luciferase activity after transient transfection of an approximately $1-\mathrm{kb}$ fragment, including the putative $M l k l$ transcriptional start site, into primary hepatocytes strongly increased upon IFN- $\gamma$ stimulation (Figure 8F). Similar results were obtained in IFN- $\gamma-$ treated human embryonic kidney cells, BNL cells, and mouse embryonic fibroblasts (MEFs) (Supplemental Figure 7F and data not shown). Moreover, ChIP assays and quantitative ChIP using a pSTAT1-specific antibody for precipitation identified STAT1 binding to predicted $\gamma$-activated sequences in the $M l k l$ promoter region in IFN- $\gamma$-stimulated BNL liver cells (Figure 8G). Binding of STAT1 to a previously described site in the Irf1 promoter served as a positive control (38) (Figure 8G). In summary, these data suggest that the proinflammatory cytokine IFN- $\gamma$ directly regulates $M l k l$ gene transcription via activation of STAT1.

Notably, while previous studies implicated the IFN- $\gamma /$ STAT1 pathway in provoking hepatocellular death by induction of apoptosis (25), our data support the notion that this pathway promotes MLKL-mediated programmed necrosis rather than apoptosis.

Having shown that IFN- $\gamma$, the sole member of the type II IFNs, induced $M l k l$ gene transcription in hepatocytes, we next analyzed the capacity of type I and type III IFNs to regulate MLKL. Thus, we next tested the contribution of type I and type III IFNs to regulation of MLKL during ConA-induced liver injury. In contrast

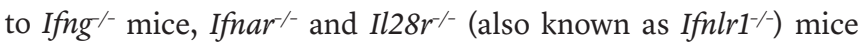
showed a comparable increase in hepatic $M l k l$ mRNA compared with wild-type controls, suggesting that, indeed, IFN- $\gamma$ is the key driver for $M l k l$ gene expression during these experimental conditions (Supplemental Figure 7G). However, in primary hepatocytes the type I IFN IFN- $\beta$ strongly increased hepatic $M l k l$ transcripts in a STAT1-dependent manner in vitro, whereas type III IFN (IFN- $\lambda$ ) stimulation did not upregulate $M l k l$ (Supplemental Figure 7, H and I). Likewise, IFN- $\beta$ treatment increased hepatic $M l k l$ mRNA in vivo (Supplemental Figure 7J), and IFN- $\beta$ stimulation of murine BNL liver cells (Supplemental Figure 7K) or MEFs (data not shown) resulted in increased $M l k l$ mRNA abundance, indicating that induction of MLKL expression may be a more general feature of IFN signaling. As IFN treatment is clinically important in several human diseases, this observation may be highly relevant for improving such therapeutic strategies. Notably, Ripk1 gene transcription was not regulated by IFN-dependent STAT1 activation (Supplemental Figure 8).

Contribution of TNF- $\alpha$ signaling to MLKL-mediated hepatocellular death. While IFN- $\gamma$ in vivo overexpression profoundly increased ConA-induced liver injury (Supplemental Figure 9, A and B), IFN- $\gamma$ alone was not sufficient to rapidly induce pronounced hepatocellular death (data not shown). Consistent with this, treatment of primary hepatocytes with IFN- $\gamma$ did not affect LDH release in vitro (Supplemental Figure 9C), suggesting that, in addition to IFN- $\gamma$ dependent MLKL upregulation, further signals are required for induction of inflammation-dependent hepatocellular death. In addition to IFN- $\gamma$, release of the proinflammatory cytokine TNF- $\alpha$ has been identified as an important contributor to liver injury (39). Given the well-known role of TNF- $\alpha$ in regulated necrosis, we therefore next addressed the role of this factor in the context of hepatic MLKL and RIPK1 activation. Although Tnfa transcripts were strongly induced in the course of ConA challenge and $\mathrm{TnFr}^{-/-}$ mice displayed protection, confirming previously published data (40) (Supplemental Figure 10, A and B, and data not shown), hepatic Mlkl and Ripk1 expression was comparable between ConAchallenged control and Tnfr $1^{-/-}$mice (Supplemental Figure 8E and Supplemental Figure 10, C and D), indicating that TNF- $\alpha$ signaling is not required for induction of $M l k l$ or Ripk1 gene transcription. By contrast, RIPK1 foci formation and MLKL translocation to membranous cellular compartments were profoundly reduced in ConA-treated $\mathrm{Tnfr}^{-1-}$ mice as compared with controls (Supplemental Figure 10, E and F). In summary, these data implicate TNF- $\alpha$ in MLKL activation during experimentally induced hepatitis. Thus, IFN- $\gamma$ and TNF- $\alpha$ synergistically drive inflammation-dependent hepatocellular death by regulation of MLKL transcription and its subsequent activation (Supplemental Figure 11).

\section{Discussion}

Irrespective of the cause of liver injury, hepatocellular death is a pathophysiologically important feature of many acute and chronic liver diseases (15). In some liver disorders with a strong inflammatory component, such as $\mathrm{AIH}$, destruction of the liver parenchyma by cellular or soluble immune factors is a central trigger of disease manifestation and progression. However, multiple signaling pathways are activated during hepatic inflammation, and so far precise therapeutically relevant mechanistic knowledge of the cell death machinery in hepatic diseases is limited. Here, we provide compelling evidence that the pseudokinase MLKL is an essential mediator of inflammation-driven hepatocellular necrosis in mice. By contrast, the activity of RIPK3 was not required for MLKLdirected death of hepatocytes in this setting, indicating the presence of a previously undescribed form of MLKL activation that may be physiologically relevant in liver diseases. On a molecular level, we further unraveled that the highly increased $M l k l$ gene expression in hepatocytes during inflammation was linked to STAT1 activation 
by the proinflammatory and cytotoxic cytokine IFN- $\gamma$, suggesting that IFN signaling-dependent regulation of MLKL is an important mechanism of sensitization of cells to regulated necrosis.

We demonstrate that elevated levels of MLKL mRNA and protein in hepatocytes relate to hepatic injury both during experimentally induced hepatitis and AIH in humans. Importantly, its upregulation in hepatitis clearly correlated to strong MLKL translocation to the plasma membrane and other membranous compartments, indicating that MLKL-dependent disruption of cellular integrity contributes to inflammation-dependent death of hepatocytes. In current models of necroptosis, MLKL plasma membrane translocation is driven by RIPK3-dependent phosphorylation (10, 11, 16, 41, 42). Importantly, while we observed a strong attenuation of acute liver damage in $\mathrm{Mlkl}^{-/-}$mice, our data provided several lines of evidence that, during experimental hepatitis, MLKL activation and translocation to membranous compartments occurs independently of RIPK3. Consistently, Ripk3 ${ }^{-/-}$mice developed ConA-induced hepatitis as severe as control mice. Accordingly, we observed only low basal levels of RIPK3 in hepatocytes, and similar to other reports $(19,43)$, RIPK3 positivity was rather limited to nonparenchymal cells, particularly Kupffer cells. Although we have previously shown that RIPK3 is upregulated in intestinal epithelial cells undergoing TNF- $\alpha$-triggered necroptosis (8), we were unable to detect induction of RIPK3 mRNA or protein in hepatocytes during the course of experimentally induced hepatitis or in human AIH. In line with this, a recent study demonstrated that RIPK3 expression in hepatocytes is dispensable for inflammatory liver injury (43).

While our data unexpectedly identified a RIPK3-independent function of MLKL, RIPK1 was not dispensable for ConAinduced hepatocellular death. A pharmacological approach to block RIPK1 kinase function was sufficient to markedly attenuate ConA-induced liver injury and to block translocation of MLKL to the plasma membrane. In line with previous reports (43), inhibition of RIPK1 kinase activity did not markedly influence immune cellmediated cytokine production. These results clearly implicate that hepatocyte-intrinsic RIPK1 kinase activity is required for MLKL plasma membrane translocation. In summary, these data implicate that RIPK1 and RIPK3 display divergent roles during hepatocellular injury and that both RIP kinases may be, independently of each other, members of cell death-related functional platforms. Further studies will have to provide more detailed information about the composition of MLKL-containing complexes, as the current notion that MLKL is solely activated by RIPK3-mediated phosphorylation is evidently not ubiquitous to all cellular contexts. This is supported by studies investigating the evolutionary origin of necroptosis that uncovered that RIPK3 is absent in many organisms, such as birds and in mammals of the superorder Xenarthra and in the Monotremata, whereas MLKL is present and RIPK1 retains its RHIM domain $(44,45)$. Interestingly, one of the phosphorylation sites in MLKL identified thus far and the intrinsic membrane-permeabilization function of MLKL seem to be conserved, even in species that have lost RIPK3, indicating that other kinases, such as RIPK1, are likely to be additional modulators of MLKL activation $(44,46)$. However, in our study we did not observe that RIPK1 directly phosphorylates MLKL during ConA hepatitis, indicating that, under these experimental conditions, an as-yet-unidentified kinase is likely to activate MLKL by phosphorylation and promote MLKL oligomerization, membrane translocation, and permeabilization. Moreover, additional phosphorylation sites in the MLKL protein might control its activation and subsequent programmed necrosis independent of RIPK3 (28). As a result, the responsible kinases and mechanisms underlying MLKL regulation by phosphorylation at these additional sites remain matters of ongoing interest.

In summary, these results provide a rationale for investigation of MLKL as a possible diagnostic biomarker for hepatocellular necrosis in $\mathrm{AIH}$ and other inflammation-dependent liver diseases. In addition, our findings support the concept that specific modulation of the MLKL pathway may be therapeutically relevant to decrease inflammation-dependent hepatocellular death and its fatal consequences in chronic human liver disorders.

While we provided compelling evidence for an essential role of MLKL during lymphocyte-mediated hepatitis, MLKL deficiency did not ameliorate APAP-induced liver injury in mice. Although previous studies have shown that RIPK3 contributes to necrotic cell death after APAP overdose $(17,21)$, oxidative stress-related mitochondrial damage may be closely related to death of hepatocytes in this model (47). This indicates that in hepatocytes several parallel mechanisms exist to execute necrotic cell death in vivo as previously shown in vitro for a variety of other cell types (15). Whereas APAP overdose and other forms of DILI are characterized by sterile inflammation, hepatocellular death in the ConA model and potentially AIH depends to a large extent on the release of cytokines by T lymphocytes, NKT cells, and NK cells. Accordingly, we identified IFN- $\gamma$, a cytokine previously described as essential for ConA-induced hepatitis, as a critical mediator of MLKL-dependent hepatocellular death. For the first time to our knowledge, we describe that IFN- $\gamma$ signal transduction in hepatocytes, via activation of STAT1 and transactivation of the Mlkl promoter, rapidly increased the amount of MLKL transcripts and protein, which are low under steady-state conditions.

PMHs display spontaneous cell death during long-term cell culture but show rather low sensitivity to necroptosis induction by TNF- $\alpha / z V A D / S M A C$ mimetic treatment $(43,48)$. However, pretreatment of mice or cells with IFN- $\gamma$ upregulated $M l k l$ in hepatocytes and increased their susceptibility toward cell death (Supplemental Figure 12). Similarly, it was recently reported that MEFs may require IFN pretreatment for effective necroptosis induction (49), indicating that sensitization of cells with IFNs may represent a general mechanism supporting programmed necrosis. IFN-induced necroptosis was described in macrophages in the presence of caspase inhibitors. Interestingly, in this study Ifnar1 ${ }^{-/-}$ macrophages were resistant to TNF- $\alpha / z V A D-i n d u c e d$ necroptosis, indicating that feedback signaling through IFNs is essential for sustained activation of RIP kinases in response to TNF- $\alpha$, LPS, or poly(I:C) (50). Our data now provide compelling evidence that, in addition to such other cell death-promoting properties of IFNs, the transcriptional regulation of MLKL by these factors is particularly important in cells that do not express substantial basal levels of MLKL, such as hepatocytes. Thus, while previous studies implicated the IFN- $\gamma /$ STAT1 pathway in promoting hepatocellular death by induction of apoptosis (25), our data support the notion that this pathway provokes MLKL-mediated programmed necrosis of hepatocytes rather than apoptosis. These data add to the molecular understanding of IFN-mediated tissue damage 
and establish a link between proinflammatory cytokine production during inflammation and regulated necrosis beyond TNF- $\alpha-$ induced signal transduction. Of note, IFNs are not only implicated in many autoimmune diseases but broadly used in the therapy of, for example, viral hepatitis and multiple sclerosis. Therapy is commonly associated with adverse side effects, such as hepatoxicity, autoantibody production, and $\mathrm{AIH}$, and this leads to dose reductions or termination of therapy $(51,52)$. IFN-mediated induction of $M L K L$ and subsequent activation of hepatocellular necrosis may significantly contribute to these side effects, and this observation may thus be relevant for the future of IFN-related therapies. Such strategies may involve cotreatment of patients with IFNs and chemical compounds that inhibit the activity of MLKL.

Collectively, our data uncover a form of programmed necrotic cell death that is dependent on the RIPK1/MLKL axis but does not require RIPK3. Depending on the physiological context, MLKL has the capacity to promote RIPK3-mediated necroptosis or alternatively triggers IFN-mediated RIPK3-independent programmed necrosis. In addition, our results suggest a physiological relevance for IFN-triggered MLKL-dependent death in the pathogenesis of inflammation-dependent liver injury such as AIH.

\section{Methods}

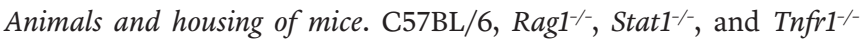
mice were obtained from The Jackson Laboratory. Ripk $3^{-1-}$ mice were described previously (53). Ifng $g^{-/-}$and Ifnar ${ }^{-/}$mice were provided by U. Schleicher. $M l k l^{--}$mice were provided by J. Murphy. Throughout, we used either C57BL/6J or littermate controls to exclude straindependent differences in disease susceptibility. Mice were routinely screened for pathogens according to FELASA guidelines.

Animal model of liver injury. LPS and GalN (catalog G1639) were obtained from Sigma-Aldrich. ConA was from Merck Millipore (cata$\log 234567)$. APAP was from Braun. Z-Val-Ala-DL-Asp-fluoromethylketone (zVAD) was from Bachem (catalog N-1510). Nec-1s was from BioVision (catalog 2263). ConA was administered intravenously (25 $\mathrm{mg} / \mathrm{kg}$ body weight) alone or in combination with nec-1s (400 $\mu$ g, i.p., 30 minutes) or zVAD ( $250 \mu \mathrm{g} /$ mouse, i.p., 15 minutes) before ConA administration. Stock solutions of nec-1s were prepared in DMSO (final concentration 0.5\%). D-GalN (1 mg/kg) was injected i.p. 1 hour prior to administration of LPS $(100 \mu \mathrm{g} / \mathrm{kg}$, i.p.). APAP $(400 \mathrm{mg} / \mathrm{kg})$ was injected i.p. after overnight fasting. Plasma concentrations of AST and ALT were measured in the clinical chemistry unit of the University Medical Center Erlangen.

For generation of bone marrow chimeras, $1 \times 10^{7}$ cells from femurs and tibias of donor mice were intravenously injected into lethally irradiated (10 Gy) recipient C57BL/6 mice. Mice were left to recover for 8 weeks before ConA was administrated.

Cell lines. HEK293T and BNL cells were obtained from ATCC. HEK293T cells, BNL cells, and MEFs were cultured in Dulbecco's Modified Eagle Medium or RPMI 1640 containing penicillin (100 U/ml, GIBCO), streptomycin (100 $\mu \mathrm{g} / \mathrm{ml}, \mathrm{GIBCO}), 10 \% \mathrm{FCS}$, L-glutamine $(200 \mathrm{mM})$, sodium pyruvate $(400 \mathrm{mM})$, and glucose $(4.5 \mathrm{~g} / \mathrm{l})$. Primary hepatocytes were isolated as described previously (54). Purified hepatocytes were seeded in collagen-coated culture plates and cultured using the following medium: William's Medium E containing $100 \mathrm{U} / \mathrm{ml}$ penicillin, $100 \mu \mathrm{g} / \mathrm{ml}$ streptomycin, $120 \mathrm{mg} / \mathrm{ml}$ gentamicin, $4 \%$ FCS, $2 \mathrm{mM}$ glutamine, $100 \mathrm{nM}$ insulin, and $100 \mathrm{nM}$ dexamethasone.
In some experiments, PMHs, BNL cells, or MEFs were stimulated with $100 \mathrm{ng} / \mathrm{ml}$ of recombinant mouse IFN- $\beta$ (R\&D Systems), $100 \mathrm{ng} /$ $\mathrm{ml}$ recombinant mouse IFN- $\gamma$ (Preprotech), $100 \mathrm{ng} / \mathrm{ml}$ recombinant mouse IL-28 (IFN- $\lambda$, R\&D Systems), $8 \mathrm{mM}$ APAP, or murine sera in a final concentration of $3 \%$. HEK293T cell were stimulated with 100 $\mathrm{ng} / \mathrm{ml}$ recombinant human IFN- $\gamma$ (Roche). For cytokine release, isolated splenocytes were cultured in RPMI 1640 supplemented with PMA (50 ng/ml, Calbiochem) in combination with ionomycin (500 $\mathrm{ng} / \mathrm{ml}$, Cayman) for 24 hours. Cell viability was measured using the Cytotoxitiy Detection Kit (LDH, Roche) or the Cell Death Detection ELISA $^{\text {PLUS }}$ Kit (Roche) accordingly to the manufacturer's instruction. Cell viability was calculated as follows: cell viability level $=1 /$ mean cell viability of the control group.

Cytokine measurements. For determination of mouse IFN- $\gamma$ or TNF- $\alpha$, specific DuoSet ELISA Kits from R\&D Systems were used. ELISA was performed according to manufacturer's instructions.

Transient transfections and luciferase assays. An approximately $1-\mathrm{kb}$ fragment, including the putative mouse $M l k l$ gene promoter sequence, was identified using the Genomatix software suite (http://www. genomatix.de) and amplified using the PfuUltra II Fusion HS DNA Polymerase (Aligent Technologies) out of tail genomic DNA using the primers $5^{\prime}$-ccctacttgggtaacttcac- $3^{\prime}$ and $5^{\prime}$-gaatgccattcctcgatctc- $3^{\prime}$. The PCR product was finally cloned upstream of the firefly luciferase open reading frame in the pGL- 4.1 vector (Promega) using the NheI and HindIII sites. For the transfection of HEK293T or PMH cells, the cells were seeded at 300,000 (HEK293T) or 500,000 (PMH) cells per 6-well plate and transfected 1 day after with $5 \mu \mathrm{g}$ DNA using Lipofectamine 2000 transfection reagent according to the manufacturer's instructions. The Luciferase Reporter Assay System from Promega was used for the analysis of luciferase activity in cell lysates according to the manufacturer's instructions.

ChIP assay. Chip assay was performed using the SimpleChIP Enzymatic Chromatin IP Kit (Magnetic Beads) from Cell Signaling accordingly to the manufacturer's instruction. In brief, BNL cells were stimulated with IFN- $\gamma$ for 45 minutes, fixed by addition of $1 \%$ formaldehyde, lysed, and treated with 1,200 U Micrococcus nuclease. Protein-DNA complexes were immunoprecipitated overnight using anti-pSTAT1 ${ }^{\text {Tyr701 }}$ (Cell Signaling, catalog 9167) or control antibodies (Cell Signaling, catalog 2729) and protein A-coupled magnetic beads. Real-time PCR on silica column-purified DNA was performed using the following primers: STAT1-Mlkl $5^{\prime}$-gaactgagcgatagac- $3^{\prime}$ and $5^{\prime}$-cgaaaccaacctaag- $3^{\prime}$ or STAT1-Irf1 $5^{\prime}$-agcacagctgacttcc-3' and 5'-cttagactgtacgtcc-3' Quantification of DNA by qPCR was performed as follows: percentage input $=$ $2 \% \times 2(\mathrm{C}[\mathrm{T}] 2 \%$ input sample $-\mathrm{C}[\mathrm{T}] \mathrm{IP}$ sample), where $\mathrm{C}[\mathrm{T}]$ denotes the threshold cycle of the PCR reaction.

IP and immunoblotting. Proteins were isolated from liver biopsies using Cell lysis buffer (Cell Signaling) supplemented with $1 \mathrm{mM}$ PMSF (Cell Signaling). Isolation of plasma membrane proteins was performed using the Plasma Membrane Protein Extraction Kit (catalog ab65400, abcam) accordingly to the manufacturer's instructions. Lysates were then centrifuged at $18,000 \mathrm{~g}$ for 20 minutes. For IP experiments supernatants were precleared and subsequently immunoprecipitated with anti-MLKL antibody (1:50, Merck Millipore, clone 3H1) at $4^{\circ} \mathrm{C}$ overnight. Subsequently, lysates were further incubated with protein $\mathrm{G}^{-}$ coupled magnetic beads for 20 minutes at room temperature. After IP, the beads were washed 4 times with lysis buffer, and the immunoprecipitated proteins were subsequently eluted with SDS sample buffer. 
Proteins were separated using a MiniProtean-TGX gel (4\%-15\% polyacrylamide; Bio-Rad) and transferred from the gel to a nitrocellulose membrane (Whatman). Membranes were probed with the following primary antibodies: murine MLKL (Biorbyt, catalog orb32399); RIPK1 (catalog 3493), Na, K-ATPase (catalog 3010), pSTAT1 (catalog 9167), and STAT1 (catalog 14994) (all from Cell Signalling); and murine RIPK3 (catalog ab62344), GAPDH (catalog ab9482), and ACTIN (catalog ab49900) (Abcam). HRP-linked anti-rabbit (catalog 7074) (Cell Signaling) was used as a secondary antibody.

Liver-specific vectors and hydrodynamic tail vein injection. The generation of vectors encoding secreted version of IFNs controlled by liver-specific regulatory elements was described previously (55). Sustained in vivo transduction of hepatocytes with IFNs was achieved by hydrodynamic delivery of $10 \mu \mathrm{g}$ vector in ringer solution $(10 \%$ of body weight). Overexpression of IFNs in hepatocytes was confirmed by ELISA. Mice injected with empty control vector served as controls.

Histology and immunohistochemistry. Histopathological analyses were performed on formalin-fixed paraffin-embedded tissue after Mayer's H\&E staining. Immunofluorescence of tissue sections was performed using the TSA Cy3/Fluorescein system, as recommended by the manufacturer (Perkin Elmer). The following antibodies were used: murine MLKL (Biorbyt, catalog orb32399); $\beta$-catenin (catalog 8480), human MLKL (catalog 14993), human pMLKL (catalog 14516), RIPK1 (catalog 3493), and cleaved caspase-3 (catalog 9661) (all from Cell Signaling); murine RIPK3 (catalog ab62344), albumin (catalog ab106582), and anti-chicken (catalog ab150169) (all from Abcam); F4/80 (AbD Serotec, catalog MCA497); and anti-rabbit (Dianova, catalog 111-065-144). Nuclei were counterstained with Hoechst 3342 (Invitrogen). Cell death (TUNEL) was analyzed using the In Situ Cell Death Detection Kit (Roche). Murine RIPK1 expression was analyzed using the Envision ${ }^{+}$system (Dako). Images were obtained using a confocal fluorescence microscope (LEICA TCS SP5 II) or the microscope LEICA DMI 4000B, together with the LEICA DFC360 FX or LEICA DFC420 C camera and the imaging software "LAS AF" (Leica).

Gene expression. Total RNA was extracted from liver tissue using the peqGOLD Total RNA Kit and from cells using the peqGOLD Microspin Total RNA Kit (Peqlab). cDNA was synthesized using the SCRIPT cDNA Synthesis Kit from Jena Bioscience and analyzed by real-time PCR using specific QuantiTect Primer assays (Qiagen). Experimental values were normalized to levels of the housekeeping gene hypoxanthine guanine phosphoribosyl transferase (Hprt).

Recombinant protein expression and purification. cDNAs encoding human RIPK1 kinase domain (residues 2-324) and human MLKL (residues 2-471) were both synthesized by DNA2.0 (CA) and ligated as BamHI-EcoRI fragments into pFastBac GST. A cDNA encoding human RIPK3 (residues 2-356) was amplified by PCR and ligated as a BamHINotI fragment into pFastBac HTb (Life Technologies). All insert sequences were verified by Sanger sequencing (Micromon, Monash University, Melbourne, Australia). Bacmids and baculoviruses were generated as previously described $(56,57)$. Recombinant proteins were expressed and purified from $S f 21$ insect cells cultured in InsectExpress media (LONZA). Purifications were performed using standard affinity chromatography and size exclusion chromatography protocols. hRIPK1 and hMLKL were purified via an N-terminal GST-tag, as described previously for hMLKL (46), and hRIPK3 was purified as described previously for His -tagged proteins (56). In all cases, affinity tags were cleaved using TEV protease and the proteins eluted from Super- dex-200 gel filtration columns (GE Healthcare) in $200 \mathrm{mM} \mathrm{NaCl}, 20$ mM HEPES, pH 7.5, 5\% glycerol; concentrated by centrifugal ultrafiltration to $6.5-7 \mathrm{mg} / \mathrm{ml}$; aliquoted; and snap frozen in liquid $\mathrm{N}_{2}$, before storage at $-80^{\circ} \mathrm{C}$ until required.

In vitro kinase assays on purified proteins. Kinase assays for purified recombinant kinase domains of hRIPK1 and hRIPK3 in the presence or absence of recombinant human MLKL were performed essentially as described previously $(31,58)$. Briefly, $3 \mu \mathrm{g}$ recombinant hMLKL was incubated in the absence or presence of $100 \mathrm{ng}$ of recombinant hRIPK1 or hRIPK3 for 5 minutes at $25^{\circ} \mathrm{C}$ after the reaction was initiated by addition of $5 \mu \mathrm{Ci}{ }^{32} \mathrm{P}-\gamma$-ATP and $37.5 \mu \mathrm{M}$ cold ATP in a $40-\mu \mathrm{l}$ reaction. In parallel, $1 \mu \mathrm{g}$ of either hRIPK1 or hRIPK3 kinase domain was reacted analogously in the absence of substrate. Kinase reaction buffer comprised $100 \mathrm{mM} \mathrm{NaCl}, 20 \mathrm{mM}$ Tris, $\mathrm{pH}$ 8, 4 mM $\mathrm{MgCl}_{2}, 0.1$ $\mathrm{mM}$ DTT, and $1 \mathrm{mg} / \mathrm{ml} \mathrm{BSA}$. Reactions were terminated by boiling in $1 \times$ reducing SDS-PAGE loading buffer for 5 to 10 minutes before one-quarter of each reaction was resolved by $4 \%-12 \%$ Bis-Tris reducing SDS-PAGE. The gel stained with SimplyBlue SafeStain (Thermo Fisher) was dried under a vacuum and exposed to a phosphor screen for 16 hours before scanning using a Typhoon imager (GE Healthcare).

Statistics. Statistical analysis was performed using the 2-tailed Student's $t$ test. $P$ values of less than 0.05 were considered significant.

Study approval. Animal protocols were approved by the Institutional Animal Care and Use Committee of the Regierung von Unterfranken. All studies with human material were approved by the ethics committee of the University Hospital of Erlangen. The diagnosis of AIH was defined according to the guidelines of the European Association for the Study of Liver Disease (59) and was based on compatible symptoms and signs of AIH, elevated ALT and AST, increased serum IgG, serological findings (elevated antinuclear antibodies, smooth muscle antibody, soluble liver antigen, or liver kidney/microsome antibodies), and histological findings, such as interface hepatitis and plasma cell infiltration. The acute forms of DILI can be divided into hepatocellular, cholestatic, and mixed forms. In this study cohort of DILI patients, all cases presented mixed forms indicated by the presence of hepatocellular and cholangiocellular damage. The extent of hepatocellular and cholangiocellular toxicity was variable but never purely hepatocellular or cholestatic. Other conditions that may cause hepatitis, including viral, drug-induced, cholestatic, metabolic, and hereditary disorders, have been excluded in these patients.

\section{Author contributions}

CG, SW, and CB designed the research. CG, GWH, JMM, and EJP performed the experiments. AEK, KA, PV, AL, CP, CD, SK, US, and MFN supplied materials that made this study possible. CG, $\mathrm{CB}$, and SW analyzed the data and wrote the paper. PV, JMM, and SK gave feedback on the draft paper.

\section{Acknowledgments}

The authors thank C. Lindner, G. Förtsch, A. Taut, S. Wallmüller, H. Dorner, and V. Thonn for excellent technical assistance. We thank W. Alexander for providing the $\mathrm{Mlkl}^{-/}$mice. We thank N. Takahashi and Y. Dondelinger for providing materials and S. Zopf for sampling of liver biopsies. We thank Sam Young for assistance with preparing recombinant protein expression constructs and bacmids. This research has received funding from DFG projects within SFB796, SPP1656, SFB1181-A08, project BE3686/2, 
KR4391/1-1, and the clinical research unit KFO257. Further support was given by the Interdisciplinary Center for Clinical Research of the University Erlangen-Nuremberg. JMM acknowledges support from the National Health and Medical Research Council of Australia (project 1057905, fellowship 1105754, and Independent Research Institute Infrastructure Support Scheme, 9000220) and the Victorian Government Operational Infrastruc- ture Support Scheme. SK acknowledges support from Dr. Werner Jackstädt-Stiftung and Fresenius Medical Care Germany.

Address correspondence to: Stefan Wirtz, Medical Department 1, University of Erlangen-Nuremberg_Hartmannstrasse 14, 91052 Erlangen, Germany. Phone: 49.0.9131.85.35882; E-mail: stefan. wirtz@uk-erlangen.de.
1. Vandenabeele P, Galluzzi L, Vanden Berghe T, Kroemer G. Molecular mechanisms of necroptosis: an ordered cellular explosion. Nat Rev Mol Cell Biol. 2010;11(10):700-714.

2. Degterev A, et al. Chemical inhibitor of nonapoptotic cell death with therapeutic potential for ischemic brain injury. Nat Chem Biol. 2005;1(2):112-119.

3. Vercammen D, et al. Dual signaling of the Fas receptor: initiation of both apoptotic and necrotic cell death pathways. J Exp Med. 1998;188(5):919-930.

4. Zhang H, Zhou X, McQuade T, Li J, Chan FK, Zhang J. Functional complementation between FADD and RIP1 in embryos and lymphocytes. Nature. 2011;471(7338):373-376.

5. Kaiser WJ, et al. RIP3 mediates the embryonic lethality of caspase-8-deficient mice. Nature. 2011;471(7338):368-372.

6. Oberst A, et al. Catalytic activity of the caspase8-FLIP(L) complex inhibits RIPK3-dependent necrosis. Nature. 2011;471(7338):363-367.

7. Sun L, et al. Mixed lineage kinase domain-like protein mediates necrosis signaling downstream of RIP3 kinase. Cell. 2012;148(1-2):213-227.

8. Günther C, et al. Caspase-8 regulates TNF- $\alpha$ induced epithelial necroptosis and terminal ileitis. Nature. 2011;477(7364):335-339.

9. Feoktistova M, et al. cIAPs block Ripoptosome formation, a RIP1/caspase-8 containing intracellular cell death complex differentially regulated by cFLIP isoforms. Mol Cell. 2011;43(3):449-463.

10. Cai Z, et al. Plasma membrane translocation of trimerized MLKL protein is required for TNF-induced necroptosis. Nat Cell Biol. 2014;16(1):55-65.

11. Hildebrand JM, et al. Activation of the pseudokinase MLKL unleashes the four-helix bundle domain to induce membrane localization and necroptotic cell death. Proc Natl Acad Sci U S A. 2014;111(42):15072-15077.

12. Bonnet MC, et al. The adaptor protein FADD protects epidermal keratinocytes from necroptosis in vivo and prevents skin inflammation. Immunity. 2011;35(4):572-582.

13. Ma X, et al. The oncogenic microRNA miR-21 promotes regulated necrosis in mice. Nat Commun. 2015;6:7151.

14. Linkermann A, et al. Two independent pathways of regulated necrosis mediate ischemia-reperfusion injury. Proc Natl Acad Sci U S A. 2013;110(29):12024-12029.

15. Luedde T, Kaplowitz N, Schwabe RF. Cell death and cell death responses in liver disease: mechanisms and clinical relevance. Gastroenterology. 2014;147(4):765-783.e4.

16. Wang $\mathrm{H}$, et al. Mixed lineage kinase domain-like protein MLKL causes necrotic membrane dis- ruption upon phosphorylation by RIP3. Mol Cell. 2014;54(1):133-146.

17. Ramachandran A, McGill MR, Xie Y, Ni HM, Ding WX, Jaeschke H. Receptor interacting protein kinase 3 is a critical early mediator of acetaminophen-induced hepatocyte necrosis in mice. Hepatology. 2013;58(6):2099-2108.

18. Takemoto K, et al. Necrostatin-1 protects against reactive oxygen species (ROS)-induced hepatotoxicity in acetaminophen-induced acute liver failure. FEBS Open Bio. 2014;4:777-787.

19. Dara L, et al. Receptor interacting protein kinase 1 mediates murine acetaminophen toxicity independent of the necrosome and not through necroptosis. Hepatology. 2015;62(6):1847-1857.

20. Roychowdhury S, McMullen MR, Pisano SG, Liu X, Nagy LE. Absence of receptor interacting protein kinase 3 prevents ethanol-induced liver injury. Hepatology. 2013;57(5):1773-1783.

21. Deutsch M, et al. Divergent effects of RIP1 or RIP3 blockade in murine models of acute liver injury. Cell Death Dis. 2015;6:e1759.

22. Manns MP, Lohse AW, Vergani D. Autoimmune hepatitis--Update 2015. J Hepatol. 2015;62(1 Suppl):S100-S111.

23. Hussain MJ, Mustafa A, Gallati H, Mowat AP, Mieli-Vergani G, Vergani D. Cellular expression of tumour necrosis factor-alpha and interferon-gamma in the liver biopsies of children with chronic liver disease. J Hepatol. 1994;21(5):816-821.

24. Küsters S, Gantner F, Künstle G, Tiegs G. Interferon gamma plays a critical role in T cell-dependent liver injury in mice initiated by concanavalin A. Gastroenterology. 1996;111(2):462-471.

25. Horras CJ, Lamb CL, Mitchell KA. Regulation of hepatocyte fate by interferon- $\gamma$. Cytokine Growth Factor Rev. 2011;22(1):35-43.

26. Siebler J, et al. A key pathogenic role for the STAT1/T-bet signaling pathway in T-cellmediated liver inflammation. Hepatology. 2003;38(6):1573-1580.

27. Sun R, et al. STAT1 contributes to dsRNA inhibition of liver regeneration after partial hepatectomy in mice. Hepatology. 2006;44(4):955-966.

28. Tanzer MC, et al. Necroptosis signalling is tuned by phosphorylation of MLKL residues outside the pseudokinase domain activation loop. Biochem J. 2015;471(2):255-265.

29. Tiegs G, Hentschel J, Wendel A. A T cell-dependent experimental liver injury in mice inducible by concanavalin A. JClin Invest. 1992;90(1):196-203.

30. Heymann F, Hamesch K, Weiskirchen R, Tacke $\mathrm{F}$. The concanavalin A model of acute hepatitis in mice. Lab Anim. 2015;49(1 Suppl):12-20.

31. Murphy JM, et al. The pseudokinase MLKL mediates necroptosis via a molecular switch mechanism. Immunity. 2013;39(3):443-453.

32. Degterev A, et al. Identification of RIP1 kinase as a specific cellular target of necrostatins. Nat Chem Biol. 2008;4(5):313-321.

33. Dillon CP, et al. RIPK1 blocks early postnatal lethality mediated by caspase- 8 and RIPK3. Cell. 2014;157(5):1189-1202.

34. Rickard JA, et al. RIPK1 regulates RIPK3-MLKLdriven systemic inflammation and emergency hematopoiesis. Cell. 2014;157(5):1175-1188.

35. Kaiser WJ, et al. RIP1 suppresses innate immune necrotic as well as apoptotic cell death during mammalian parturition. Proc Natl Acad Sci US A. 2014;111(21):7753-7758.

36. Duprez L, et al. RIP kinase-dependent necrosis drives lethal systemic inflammatory response syndrome. Immunity. 2011;35(6):908-918.

37. Rodriguez DA, et al. Characterization of RIPK3-mediated phosphorylation of the activation loop of MLKL during necroptosis. Cell Death Differ. 2016;23(1):76-88.

38. Pine R, Canova A, Schindler C. Tyrosine phosphorylated p91 binds to a single element in the ISGF2/ IRF-1 promoter to mediate induction by IFN alpha and IFN gamma, and is likely to autoregulate the p91 gene. EMBO J. 1994;13(1):158-167.

39. Maeda S, Chang L, Li ZW, Luo JL, Leffert H, Karin M. IKKbeta is required for prevention of apoptosis mediated by cell-bound but not by circulating TNFalpha. Immunity. 2003;19(5):725-737.

40. Küsters S, et al. In vivo evidence for a functional role of both tumor necrosis factor (TNF) receptors and transmembrane TNF in experimental hepatitis. Eur J Immunol. 1997;27(11):2870-2875.

41. Chen X, et al. Translocation of mixed lineage kinase domain-like protein to plasma membrane leads to necrotic cell death. Cell Res. 2014;24(1):105-121.

42. Dondelinger $Y$, et al. MLKL compromises plasma membrane integrity by binding to phosphatidylinositol phosphates. Cell Rep. 2014;7(4):971-981.

43. Kang YJ, et al. Regulation of NKT cell-mediated immune responses to tumours and liver inflammation by mitochondrial PGAM5-Drp1 signalling. Nat Commun. 2015;6:8371.

44. Newton K, Manning G. Necroptosis and inflammation. Annu Rev Biochem. 2016;85:743-763.

45. Dondelinger Y, Hulpiau P, Saeys Y, Bertrand MJ, Vandenabeele P. An evolutionary perspective on the necroptotic pathway [published online ahead of print June 28, 2016]. Trends Cell Biol. doi: 10.1016/j.tcb.2016.06.004.

46. Tanzer MC, et al. Evolutionary divergence of the necroptosis effector MLKL. Cell Death Differ. 2016;23(7):1185-1197.

47. McGill MR, Sharpe MR, Williams CD, Taha M, Curry SC, Jaeschke H. The mechanism underlying acetaminophen-induced hepatotoxicity in humans and mice involves mitochondrial 


\section{RESEARCH ARTICLE}

damage and nuclear DNA fragmentation. J Clin Invest. 2012;122(4):1574-1583.

48. Vinken M, et al. Primary hepatocytes and their cultures in liver apoptosis research. Arch Toxicol. 2014;88(2):199-212.

49. Rodriguez DA, et al. Characterization of RIPK3-mediated phosphorylation of the activation loop of MLKL during necroptosis. Cell Death Differ. 2016;23(1):76-88.

50. McComb S, et al. Type-I interferon signaling through ISGF3 complex is required for sustained Rip3 activation and necroptosis in macrophages. Proc Natl Acad Sci U S A. 2014;111(31):E3206-E3213.

51. Dusheiko G. Side effects of alpha interferon in chronic hepatitis C. Hepatology. 1997; 26(3 Suppl 1):112S-121S.

52. García-Buey L, et al. Latent autoimmune hepati- tis triggered during interferon therapy in patients with chronic hepatitis C. Gastroenterology. 1995;108(6):1770-1777.

53. Newton K, Sun X, Dixit VM. Kinase RIP3 is dispensable for normal NF-kappa Bs, signaling by the B-cell and T-cell receptors, tumor necrosis factor receptor 1, and Toll-like receptors 2 and 4 . Mol Cell Biol. 2004;24(4):1464-1469.

54. Bajt ML, Knight TR, Lemasters JJ, Jaeschke H. Acetaminophen-induced oxidant stress and cell injury in cultured mouse hepatocytes: protection by $\mathrm{N}$-acetyl cysteine. Toxicol Sci. 2004;80(2):343-349.

55. McHedlidze T, et al. Interleukin-33-dependent innate lymphoid cells mediate hepatic fibrosis. Immunity. 2013;39(2):357-371.

56. Murphy JM, et al. A robust methodology to subclassify pseudokinases based on their nucleotide-bind- ing properties. Biochem J. 2014;457(2):323-334.

57. Babon JJ, Murphy JM. In vitro JAK kinase activity and inhibition assays. Methods Mol Biol. 2013;967:39-55.

58. Cook WD, et al. RIPK1- and RIPK3-induced cell death mode is determined by target availability. Cell Death Differ. 2014;21(10):1600-1612.

59. European Association for the Study of the Liver. EASL Clinical Practice Guidelines: Autoimmune hepatitis. J Hepatol. 2015;63(4):971-1004.

60. Cartharius K, et al. MatInspector and beyond: promoter analysis based on transcription factor binding sites. Bioinformatics. 2005;21(13):2933-2942.

61. Marinescu VD, Kohane IS, Riva A. MAPPER: a search engine for the computational identification of putative transcription factor binding sites in multiple genomes. BMC Bioinformatics. 2005;6:79. 